\title{
Information-Based Asset Pricing
}

\author{
Dorje C. Brody*, Lane P. Hughston ${ }^{\dagger}$, and Andrea Macrina ${ }^{\dagger}$ \\ *Blackett Laboratory, Imperial College, London SW7 2BZ, UK \\ $\dagger$ Department of Mathematics, King's College London, \\ The Strand, London WC2R 2LS, UK
}

\begin{abstract}
A new framework for asset price dynamics is introduced in which the concept of noisy information about future cash flows is used to derive the corresponding price processes. In this framework an asset is defined by its cash-flow structure. Each cash flow is modelled by a random variable that can be expressed as a function of a collection of independent random variables called market factors. With each such " $X$-factor" we associate a market information process, the values of which we assume are accessible to market participants. Each information process consists of a sum of two terms; one contains true information about the value of the associated market factor, and the other represents "noise". The noise term is modelled by an independent Brownian bridge that spans the interval from the present to the time at which the value of the factor is revealed. The market filtration is assumed to be that generated by the aggregate of the independent information processes. The price of an asset is given by the expectation of the discounted cash flows in the risk-neutral measure, conditional on the information provided by the market filtration. In the case where the cash flows are the dividend payments associated with equities, an explicit model is obtained for the share-price process. Dividend growth is taken into account by introducing appropriate structure on the market factors. The prices of options on dividend-paying assets are derived. Remarkably, the resulting formula for the price of a European-style call option is of the Black-Scholes-Merton type. We consider both the case where the rate at which information is revealed to the market is constant, and the case where the information rate varies in time. Option pricing formulae are obtained for both cases. The information-based framework generates a natural explanation for the origin of stochastic volatility in financial markets, without the need for specifying on an ad hoc basis the dynamics of the volatility.
\end{abstract}

Key words: Asset pricing; partial information; stochastic volatility; correlation; dividend growth; Brownian bridge; nonlinear filtering; market microstructure

Working paper. Original version: December 5, 2005. This version: October 22, 2018.

Email: dorje@imperial.ac.uk, lane.hughston@kcl.ac.uk, andrea.macrina@kcl.ac.uk

\section{INTRODUCTION}

In derivative pricing, the starting point is usually the specification of a model for the price process of the underlying asset. Such models tend to be of an ad hoc nature. For example, in the Black-Scholes-Merton-Merton theory, the underlying asset has a geometric 
Brownian motion as its price process. More generally, the economy is often modelled by a probability space equipped with the filtration generated by a multi-dimensional Brownian motion, and it is assumed that asset prices are adapted to this filtration. This example is of course the "standard" model within which a great deal of financial engineering has been carried out. The basic problem with the standard model (and the same applies to various generalisations thereof) is that the market filtration is fixed, and no comment is offered on the issue of "where it comes from". In other words, the filtration, which represents the revelation of information to market participants, is modelled first, in an ad hoc manner, and then it is assumed that the asset price processes are adapted to it. But no indication is given about the nature of this "information", and it is not obvious, a priori, why the Brownian filtration, for example, should be regarded as providing information rather than noise.

In a complete market there is a sense in which the Brownian filtration provides no irrelevant information. That is to say, in a complete market based on a Brownian filtration the asset price movements reflect the information content of the filtration. Nevertheless, the notion that the market filtration should be "prespecified" is an unsatisfactory one in financial modelling. The intuition behind the "prespecified-filtration" approach is that the filtration represents the unfolding in time of a succession of random events that "influence" the markets, causing prices to change. For example, bad weather in South America results in a decrease in the supply of coffee beans and hence an increase in the price of coffee. Or, say, a spate of bad derivative deals causes a drop in confidence in banks, and hence a downgrade in earnings projections, and thus a drop in their prices. The idea is that one "abstractifies" these influences in the form of a prespecified background filtration to which price processes are adapted. What is unsatisfactory about this is that little structure is given to the filtration: price movements behave as though they were spontaneous. In reality, we expect the price-formation process to exhibit more structure.

It would be out of place in the present context to attempt an account of the process of price formation. Nevertheless, we can improve on the "prespecified" approach. In that spirit we proceed as follows. We note that price changes arise from two sources. The first is that resulting from changes in agent preferences - that is to say, changes in the pricing kernel. Movements in the pricing kernel are associated with (a) changes in investor attitudes towards risk, and (b) changes in investor "impatience", the subjective discounting of future cash flows. Equally important are changes in price resulting from the revelation of information about the future cash flows derivable from a given asset. When a market agent decides to buy or sell an asset, the decision is made in accordance with the information available to the agent concerning the likely future cash flows associated with the asset. A change in the information available to the agent about a future cash flow will typically have an effect on the price at which they are willing to buy or sell, even if the agent's preferences remain unchanged. Consider the situation where one is thinking of purchasing an item at a price that seems attractive. But then, one reads an article pointing out an undesirable feature of the product. After reflection, one decides that the price is too high, given the deficiencies that one is now aware of. As a result, one decides not to buy, not at that price, and eventually - because other individuals will have read the same report - the price drops.

The movement of the price of an asset should, therefore, be regarded as an emergent phenomenon. To put the matter another way, the price process of an asset should be viewed as the output of (rather than an input into) the decisions made relating to possible transactions in the asset, and these decisions should be understood as being induced primarily by the flow of information to market participants. Taking into account this observation we 
propose in this paper a new framework for asset pricing based on modelling of the flow of market information. The information is that concerning the values of the future cash flows associated with the given assets. For example, if the asset represents a share in a firm that will make a single distribution at some agreed date, then there is a single cash flow. If the asset is a credit-risky discount bond, then the future cash flow is the payout of the bond at maturity. In each case, based on the information available relating to the likely payouts of the given financial instrument, market participants determine estimates for the value of the right to the impending cash flows. These estimates lead to the decisions concerning transactions that trigger movements in the price.

In this paper we present a class of models capturing the essence of the scenario described above. In building the framework we have several criteria in mind that we would like to see satisfied. The first of these is that our model for the flow of market information should be intuitively appealing, and should allow for a reasonably sophisticated account of aggregate investor behaviour. At the same time, the model should be simple enough to allow one to derive explicit expressions for the asset price processes thus induced, in a suitably rich range of examples, as well as for various associated derivative price processes. The framework should also be flexible enough to allow for the modelling of assets having complex cash-flow structures. Furthermore, it should be suitable for practical implementation. The framework should be mathematically sound, and manifestly arbitrage-free. In what follows we show how our modelling framework goes a long way towards satisfying these criteria.

The role of information in financial modelling has long been appreciated, particularly in the theory of market microstructure (see, e.g., Back [1], Back and Baruch [2], O'Hara [20], and references cited therein). The present framework is perhaps most closely related to the line of investigation represented, e.g., in Cetin et al. [5], Duffie and Lando [8], Giesecke [10], Giesecke and Goldberg [11], Guo et al. [13], and Jarrow and Protter [14]. The work in this paper, in particular, develops that described in Brody et al. [3] and Macrina [19], where preliminary accounts of some of this material appear (see also Rutkowski and Yu [22]).

The paper is organised as follows. In Sections II, III, and IV we illustrate the framework for information-based pricing by considering the scenario in which there is a single random cash flow. An elementary model for market information is presented, based on the specification of a process composed of two parts: a "signal" component containing true information about the upcoming cash flow, and an independent "noise" component which we model in a specific way. A closed-form expression for the asset price is obtained in terms of the market information available at the time the price is being specified. This result is summarised in Proposition 1. In Section $\nabla$ we show that the resulting asset price process is driven by a Brownian motion, an expression for which can be obtained in terms of the market information process: this construction indicates in explicit terms the sense in which the price process can be viewed as an "emergent" phenomenon. In Section VI we show that the value of a European-style call option, in the case of an asset with a single cash flow, admits a simple formula analogous to that of the Black-Scholes-Merton model. In Section VII we derive pricing formulae for the situation when the random variable associated with the single cash flow has an exponential distribution or, more generally, a gamma distribution.

The extension of the framework to assets associated with multiple cash flows is established in Section VIII. We show that once the relevant cash flows are decomposed in terms of a collection of independent market factors, then a closed-form expression for the asset price associated with a complex cash-flow structure can be obtained. In Section [X] we show how the standard geometric Brownian motion model can be derived in an information-based 
setting. This remarkable result motivates the specific choice of information process given in equation (44). In Section $\mathrm{X}$ we present a simple model for dividend growth. In Section XI we show that by allowing distinct assets to share one or more common market factors in the determination of one or more of their respective cash flows we obtain a natural correlation structure for the associated asset price processes. This method for introducing correlation in asset price movements contrasts with the ad hoc approach adopted in most financial modelling. In Section XII we demonstrate that if two or more market factors affect the future cash flows of an asset, then the corresponding price process will exhibit unhedgeable stochastic volatility. This result is noteworthy since even for the class of relatively simple models considered here it is possible to identify a candidate for the origin of stochasticity in price volatility, as well as the form it should take, which is given in Proposition 2.

In the remaining sections of the paper we consider the case where the rate at which the information concerning the true value of an impending cash flow is revealed is time dependent. The introduction of a time-dependent information rate adds additional flexibility to the modelling framework, and opens the door to the possibility of calibrating the model to the market prices of options. We consider the single-factor case first, and obtain a closedform expression for the conditional expectation of the cash flow. The result is stated first in Section XIII as Proposition 3, and the derivation is then given in the two sections that follow. In Section XIV we introduce a new measure appropriate for the consideration of a Brownian bridge with a random drift, which is used in Section XV to obtain an expression for the conditional probability density of the random cash flow. The consistency of the resulting price process is established in Section XVI. We show, in particular, that, for the given information process, if we re-initialise the model at some specified future time, the dynamics of the model moving forward from that time can be represented by a suitably re-initialised information process. The statement of this result is given in Proposition 4 . The dynamical equation satisfied by the price process is analysed in Section XVII, where we demonstrate in Proposition 5 that the driving process is a Brownian motion, just as in the constant parameter case. In Section XVIII we derive the price of a European-style call option in the case for which the information rate is time dependent.

Our approach is based on the idea that first one models the cash flows, then the information processes, then the filtration, and finally the prices. In Section XIX, we solve the corresponding "inverse" problem. The result is stated in Proposition 6. Starting from the dynamics of the conditional distribution of the impending payoff, which is driven by a Brownian motion adapted to the market filtration, we construct (a) the random variable that represents the relevant market factor, and (b) an independent Brownian bridge representing irrelevant information. These two combine to generate the market filtration. We conclude in Section $\mathrm{XX}$ with a general multi-factor extension of the time-dependent setup, for which the dynamics of the resulting price processes are given in Propositions 7 and 8 .

\section{THE MODELLING FRAMEWORK}

In asset pricing we require three ingredients: (a) the cash flows, (b) the investor preferences, and (c) the flow of information to market participants. Translated into a mathematical language, these ingredients amount to the following: $\left(\mathrm{a}^{\prime}\right)$ cash flows are modelled as random variables; $\left(b^{\prime}\right)$ investor preferences are modelled with the determination of a pricing kernel; and $\left(\mathrm{c}^{\prime}\right)$ the market information flow is modelled with the specification of a filtration. As we have indicated above, asset pricing theory conventionally attaches more weight to (a) and 
(b) than to (c). In this paper we emphasise the importance of ingredient (c).

Our theory will be based on modelling the flow of information accessible to market participants concerning the future cash flows associated with a position in a financial asset. We start by introducing the notation and assumptions employed in this paper. We model the financial markets with the specification of a probability space $(\Omega, \mathcal{F}, \mathbb{Q})$ on which a filtration $\left\{\mathcal{F}_{t}\right\}_{0 \leq t<\infty}$ will be constructed. The probability measure $\mathbb{Q}$ is understood to be the risk-neutral measure, and the filtration $\left\{\mathcal{F}_{t}\right\}$ is understood to be the market filtration. All asset-price processes and other information-providing processes accessible to market participants will be adapted to $\left\{\mathcal{F}_{t}\right\}$.

Several simplifying assumptions will be made that will enable us to concentrate our efforts on the problems associated with the flow of market information. The first of these assumptions is the use of the risk-neutral measure. The "real" probability measure does not enter into the present investigation. Expectation in the measure $\mathbb{Q}$ will be denoted by $\mathbb{E}[-]$. Our second assumption is that we take the default-free system of interest rates to be deterministic. The absence of arbitrage then implies that the corresponding system of discount functions $\left\{P_{t T}\right\}_{0 \leq t \leq T<\infty}$ can be written in the form $P_{t T}=P_{0 T} / P_{0 t}$ for $t \leq T$, where $\left\{P_{0 t}\right\}_{0 \leq t<\infty}$ is the initial discount function, which we take to be part of the initial data of the model. The function $\left\{P_{0 t}\right\}_{0 \leq t<\infty}$ is assumed to be differentiable and strictly decreasing, and to satisfy $0<P_{0 t} \leq 1$ and $\lim _{t \rightarrow \infty} P_{0 t}=0$.

We also assume, for simplicity, that cash flows occur at pre-determined dates. Now clearly for some purposes we would like to allow for cash flows occurring effectively at random times - in particular, at stopping times associated with the market filtration. But in the present exposition we want to avoid the idea of a "prespecified" filtration with respect to which stopping times are defined. We take the view that the market filtration is a "derived" notion, generated by information about upcoming cash flows, and by the values of cash flows when they occur. We shall therefore regard a "randomly-timed" cash flow as being a set of random cash flows occurring at various times, and with a joint distribution function that ensures only one of these flows is non-zero. Hence in our view the ontological status of a cash flow is that its timing is definite, only the amount is random-and that cash flows occurring at different times are, by their nature, different cash flows.

\section{MODELLING THE CASH FLOWS}

First we consider the case of an asset that provides a single isolated cash flow occurring at time $T$, represented by a random variable $D_{T}$. We assume that $D_{T} \geq 0$. The value $S_{t}$ of the cash flow at any earlier time $t$ in the interval $0 \leq t<T$ is then given by the discounted conditional expectation of $D_{T}$ :

$$
S_{t}=P_{t T} \mathbb{E}\left[D_{T} \mid \mathcal{F}_{t}\right] .
$$

In this way we model the price process $\left\{S_{t}\right\}_{0 \leq t<T}$ of a limited-liability asset that pays a single dividend $D_{T}$ at time $T$. The construction of the price process here is carried out in such a way as to guarantee an arbitrage-free market if other assets are priced by the same method (see Davis [7] for a closely related point of view). We shall use the terms "cash flow" and "dividend" more or less interchangeably. If a more specific use of one of these terms is needed, then this will be evident from the context. We adopt the convention that when the dividend is paid the asset price goes "ex-dividend" immediately. Hence in the example above we have $\lim _{t \rightarrow T} S_{t}=D_{T}$ and $S_{T}=0$. 
In the case where the asset pays a sequence of dividends $D_{T_{k}}(k=1,2, \ldots, n)$ on the dates $T_{k}$ the price (for $t<T_{1}$ ) is given by

$$
S_{t}=\sum_{k=1}^{n} P_{t T_{k}} \mathbb{E}\left[D_{T_{k}} \mid \mathcal{F}_{t}\right]
$$

More generally, for all $t \geq 0$, and taking into account the ex-dividend behaviour, we have

$$
S_{t}=\sum_{k=1}^{n} \mathbb{1}_{\left\{t<T_{k}\right\}} P_{t T_{k}} \mathbb{E}\left[D_{T_{k}} \mid \mathcal{F}_{t}\right] .
$$

It will be useful if we adopt the convention that a discount bond also goes ex-dividend on its maturity date. Thus in the case of a discount bond we assume that the price of the bond is given, for dates earlier than the maturity date, by the product of the principal and the relevant discount factor. But at maturity (when the principal is paid) the value of the bond drops to zero. In the case of a coupon bond, there is likewise a downward jump in the price of the bond at the time a coupon is paid (the value lost may be captured back in the form of an "accrued interest" payment). In this way we obtain a consistent treatment of the "ex-dividend" behaviour of all of the asset price processes under consideration. With this convention it follows that price processes are right continuous with left limits.

\section{MODELLING THE INFORMATION FLOW}

Now we present a simple model for the flow of market information, following Brody et al. [3]. We consider first the case of a single distribution, occurring at time $T$, and assume that market participants have only partial information about the upcoming cash flow $D_{T}$. The information available in the market about the cash flow is assumed to be contained in a process $\left\{\xi_{t}\right\}_{0 \leq t \leq T}$ defined by:

$$
\xi_{t}=\sigma t D_{T}+\beta_{t T}
$$

We call $\left\{\xi_{t}\right\}$ the market information process. This process is composed of two parts. The term $\sigma t D_{T}$ contains the "true information" about the dividend, and grows in magnitude as $t$ increases. The process $\left\{\beta_{t T}\right\}_{0 \leq t \leq T}$ is a standard Brownian bridge over the time interval $[0, T]$. Thus $\left\{\beta_{t T}\right\}$ is Gaussian, $\beta_{0 T}=0, \beta_{T T}=0$, the random variable $\beta_{t T}$ has mean zero, and the covariance of $\beta_{s T}$ and $\beta_{t T}$ for $s \leq t$ is $s(T-t) / T$. We assume that $D_{T}$ and $\left\{\beta_{t T}\right\}$ are independent. Thus the information contained in the bridge process is "pure noise".

We assume that the market filtration $\left\{\mathcal{F}_{t}\right\}$ is generated by the market information process: $\mathcal{F}_{t}=\sigma\left(\left\{\xi_{s}\right\}_{0 \leq s \leq t}\right)$. The dividend $D_{T}$ is therefore $\mathcal{F}_{T}$-measurable, but is not $\mathcal{F}_{t}$-measurable for $t<T$. Thus the value of $D_{T}$ becomes "known" at time $T$, but not earlier. The bridge process $\left\{\beta_{t T}\right\}$ is not adapted to $\left\{\mathcal{F}_{t}\right\}$ and thus is not directly accessible to market participants. This reflects the fact that until the dividend is paid the market participants cannot distinguish the "true information" from the "noise" in the market. The introduction of the Brownian bridge models the fact that market perceptions, whether valid or not, play a role in determining asset prices. Initially, all available information is used to determine the a priori probability distribution for $D_{T}$. The parameter $\sigma$ represents the rate at which information about the true value of $D_{T}$ is revealed as time progresses. If $\sigma$ is low, the value 
of $D_{T}$ is effectively hidden until very near the time of the dividend payment; whereas if $\sigma$ is high, then the value of the cash flow is for all practical purposes revealed quickly.

In the example under consideration we have made some simplifying assumptions concerning the information structure. For instance, we assume that $\sigma$ is constant. In Section XIII, however, we consider a time-dependent information flow rate. We have also assumed that the random dividend $D_{T}$ enters directly into the structure of the information process, and enters linearly. As we shall indicate later, a more general and natural setup is to let the information process depend on a random variable $X_{T}$ which we call a "market factor"; then the dividend is regarded as a function of the market factor. This arrangement has the advantage that it easily generalises to the situation where a cash flow might depend on several independent market factors, or indeed where cash flows associated with different financial instruments have one or more factors in common.

Given the market information structure described above for a single cash flow, we proceed to construct the associated price dynamics. The price process $\left\{S_{t}\right\}$ for a share in the firm paying the specified dividend is given by formula (11). It is assumed that the a priori probability distribution of the dividend $D_{T}$ is known. This distribution is regarded as part of the initial data of the problem, which in some cases can be calibrated from knowledge of the initial price of the asset along with other price data. The general problem of how the a priori distribution is obtained is an important one - any asset pricing model has to confront this issue - which we defer for later consideration. The initial distribution is not to be understood as being "absolutely" determined, but rather represents the "best estimate" for the distribution given the data available at that time, in accordance with what one might call a Bayesian point of view. We note the fact that the information process $\left\{\xi_{t}\right\}$ is Markovian (see Brody et al. [3], and Rutkowski and Yu [22]). Making use of this property together with the fact that $D_{T}$ is $\mathcal{F}_{T}$-measurable we deduce that

$$
S_{t}=\mathbb{1}_{\{t<T\}} P_{t T} \mathbb{E}\left[D_{T} \mid \xi_{t}\right] .
$$

If the random variable $D_{T}$ that represents the payoff has a continuous distribution, then the conditional expectation in (5) can be expressed in the form

$$
\mathbb{E}\left[D_{T} \mid \xi_{t}\right]=\int_{0}^{\infty} x \pi_{t}(x) \mathrm{d} x .
$$

Here $\pi_{t}(x)$ is the conditional probability density for the random variable $D_{T}$ :

$$
\pi_{t}(x)=\frac{\mathrm{d}}{\mathrm{d} x} \mathbb{Q}\left(D_{T} \leq x \mid \xi_{t}\right) .
$$

We implicitly assume appropriate technical conditions on the distribution of the dividend that will suffice to ensure the existence of the expressions under consideration. Also, for convenience we use a notation appropriate for continuous distributions, though corresponding results can be inferred for discrete distributions, or more general distributions, by slightly modifying the stated assumptions and conclusions.

Bearing in mind these points, we note that the conditional probability density process for the dividend can be worked out by use of a form of the Bayes formula:

$$
\pi_{t}(x)=\frac{p(x) \rho\left(\xi_{t} \mid D_{T}=x\right)}{\int_{0}^{\infty} p(x) \rho\left(\xi_{t} \mid D_{T}=x\right) \mathrm{d} x} .
$$


Here $p(x)$ denotes the a priori probability density for $D_{T}$, which we assume is known as an initial condition, and $\rho\left(\xi_{t} \mid D_{T}=x\right)$ denotes the conditional density for the random variable $\xi_{t}$ given that $D_{T}=x$. Since $\beta_{t T}$ is a Gaussian random variable with mean zero and variance $t(T-t) / T$, we deduce that the conditional probability density for $\xi_{t}$ is

$$
\rho\left(\xi_{t} \mid D_{T}=x\right)=\sqrt{\frac{T}{2 \pi t(T-t)}} \exp \left(-\frac{\left(\xi_{t}-\sigma t x\right)^{2} T}{2 t(T-t)}\right) .
$$

Inserting this expression into the Bayes formula we get

$$
\pi_{t}(x)=\frac{p(x) \exp \left[\frac{T}{T-t}\left(\sigma x \xi_{t}-\frac{1}{2} \sigma^{2} x^{2} t\right)\right]}{\int_{0}^{\infty} p(x) \exp \left[\frac{T}{T-t}\left(\sigma x \xi_{t}-\frac{1}{2} \sigma^{2} x^{2} t\right)\right] \mathrm{d} x} .
$$

We thus obtain the following result for the asset price:

Proposition 1. The information-based price process $\left\{S_{t}\right\}_{0 \leq t \leq T}$ of a limited-liability asset that pays a single dividend $D_{T}$ at time $T$ with a priori distribution

$$
\mathbb{Q}\left(D_{T} \leq y\right)=\int_{0}^{y} p(x) \mathrm{d} x
$$

is given by

$$
S_{t}=\mathbb{1}_{\{t<T\}} P_{t T} \frac{\int_{0}^{\infty} x p(x) \exp \left[\frac{T}{T-t}\left(\sigma x \xi_{t}-\frac{1}{2} \sigma^{2} x^{2} t\right)\right] \mathrm{d} x}{\int_{0}^{\infty} p(x) \exp \left[\frac{T}{T-t}\left(\sigma x \xi_{t}-\frac{1}{2} \sigma^{2} x^{2} t\right)\right] \mathrm{d} x},
$$

where $\xi_{t}=\sigma t D_{T}+\beta_{t T}$ is the market information.

\section{ASSET PRICE DYNAMICS IN THE CASE OF A SINGLE CASH FLOW}

In order to analyse the properties of the price process deduced above, and to be able to compare it with other models, we need to work out the dynamics of $\left\{S_{t}\right\}$. One of the advantages of the model under consideration is that we have an explicit expression for the price at our disposal. Thus in obtaining the dynamics we need to find the stochastic differential equation of which $\left\{S_{t}\right\}$ is the solution. This turns out to be an interesting exercise because it offers some insights into what we mean by the assertion that market price dynamics should be regarded as an "emergent phenomenon". To obtain the dynamics associated with the price process $\left\{S_{t}\right\}$ of a single-dividend paying asset let us write

$$
D_{t T}=\mathbb{E}\left[D_{T} \mid \xi_{t}\right]
$$

Evidently, $D_{t T}$ can be expressed in the form $D_{t T}=D\left(\xi_{t}, t\right)$, where $D(\xi, t)$ is defined by

$$
D(\xi, t)=\frac{\int_{0}^{\infty} x p(x) \exp \left[\frac{T}{T-t}\left(\sigma x \xi-\frac{1}{2} \sigma^{2} x^{2} t\right)\right] \mathrm{d} x}{\int_{0}^{\infty} p(x) \exp \left[\frac{T}{T-t}\left(\sigma x \xi-\frac{1}{2} \sigma^{2} x^{2} t\right)\right] \mathrm{d} x} .
$$

A straightforward calculation making use of the Ito rules shows that the dynamical equation for $\left\{D_{t T}\right\}$ is given by

$$
\mathrm{d} D_{t T}=\frac{\sigma T}{T-t} V_{t}\left[\frac{1}{T-t}\left(\xi_{t}-\sigma T D_{t T}\right) \mathrm{d} t+\mathrm{d} \xi_{t}\right] .
$$


Here $V_{t}$ is the conditional variance of the dividend:

$$
V_{t}=\mathbb{E}_{t}\left[\left(D_{T}-\mathbb{E}_{t}\left[D_{T}\right]\right)^{2}\right]=\int_{0}^{\infty} x^{2} \pi_{t}(x) \mathrm{d} x-\left(\int_{0}^{\infty} x \pi_{t}(x) \mathrm{d} x\right)^{2} .
$$

Therefore, if we define a new process $\left\{W_{t}\right\}_{0 \leq t<T}$ by setting

$$
W_{t}=\xi_{t}-\int_{0}^{t} \frac{1}{T-s}\left(\sigma T D_{t T}-\xi_{s}\right) \mathrm{d} s,
$$

we find, after some rearrangement, that

$$
\mathrm{d} D_{t T}=\frac{\sigma T}{T-t} V_{t} \mathrm{~d} W_{t}
$$

For the dynamics of the asset price we thus have

$$
\mathrm{d} S_{t}=r_{t} S_{t} \mathrm{~d} t+\Gamma_{t T} \mathrm{~d} W_{t},
$$

where $r_{t}=-\mathrm{d} \ln P_{0 t} / \mathrm{d} t$ is the short rate, and the absolute price volatility $\Gamma_{t T}$ is

$$
\Gamma_{t T}=P_{t T} \frac{\sigma T}{T-t} V_{t}
$$

A slightly different way of arriving at this result is as follows. We start with the conditional probability $\pi_{t}(x)$. Then, using the notation above, for its dynamics we obtain

$$
\mathrm{d} \pi_{t}(x)=\frac{\sigma T}{T-t}\left(x-D_{t T}\right) \pi_{t}(x) \mathrm{d} W_{t} .
$$

Since according to (5) the asset price is given by

$$
S_{t}=\mathbb{1}_{\{t<T\}} P_{t T} \int_{0}^{\infty} x \pi_{t}(x) \mathrm{d} x,
$$

we can infer the dynamics of $\left\{S_{t}\right\}$ from the dynamics of the conditional probability $\left\{\pi_{t}(x)\right\}$, once we take into account formula (16) for the conditional variance.

As we shall demonstrate later, the process $\left\{W_{t}\right\}$ defined in (17) is an $\left\{\mathcal{F}_{t}\right\}$-Brownian motion. Hence from the point of view of the market it is the process $\left\{W_{t}\right\}$ that drives the asset price dynamics. In this way our framework resolves the paradoxical point of view usually adopted in financial modelling in which $\left\{W_{t}\right\}$ is regarded on the one hand as "noise", and yet on the other hand also generates the market information flow. And thus, instead of hypothesising the existence of a driving process for the dynamics of the markets, we are able from the information-based perspective to deduce the existence of such a process.

The information-flow parameter $\sigma$ determines the overall magnitude of the volatility. In fact, $\sigma$ plays a role analogous to the similarly-labelled parameter in the Black-ScholesMerton theory. Thus, we can say that the rate at which information is revealed in the market determines the magnitude of the volatility. Everything else being the same, if we increase the information-flow rate, then the market volatility will increase as well. According to this point of view, those mechanisms that one might have thought were destined to make markets more efficient - e.g., globalisation of the financial markets, reduction of trade barriers, improved communications, a robust regulatory environment, and so on - can have the effect of increasing market volatility, and hence market risk, rather than reducing it. 


\section{EUROPEAN-STYLE CALL OPTIONS}

Before we turn to the consideration of more general cash flows and market information structures, let us consider the pricing of a derivative on an asset for which the price process is governed by (19). Specifically, we consider the valuation of a European call option on such an asset, with strike price $K$, and exercisable at a fixed date $t$. The option is written on an asset that pays a single dividend $D_{T}$ at time $T>t$. The initial value of the option is

$$
C_{0}=P_{0 t} \mathbb{E}\left[\left(S_{t}-K\right)^{+}\right] .
$$

Inserting the expression for $S_{t}$ derived in the previous section into this formula, we obtain

$$
C_{0}=P_{0 t} \mathbb{E}\left[\left(P_{t T} \int_{0}^{\infty} x \pi_{t}(x) \mathrm{d} x-K\right)^{+}\right]
$$

For convenience we write the conditional probability $\pi_{t}(x)$ in the form

$$
\pi_{t}(x)=\frac{p_{t}(x)}{\int_{0}^{\infty} p_{t}(x) \mathrm{d} x}
$$

where the "unnormalised" density $p_{t}(x)$ is defined by

$$
p_{t}(x)=p(x) \exp \left[\frac{T}{T-t}\left(\sigma x \xi_{t}-\frac{1}{2} \sigma^{2} x^{2} t\right)\right] .
$$

Substituting (26) into (24) we find that the value of the option is

$$
C_{0}=P_{0 t} \mathbb{E}\left[\frac{1}{\Phi_{t}}\left(\int_{0}^{\infty}\left(P_{t T} x-K\right) p_{t}(x) \mathrm{d} x\right)^{+}\right]
$$

where

$$
\Phi_{t}=\int_{0}^{\infty} p_{t}(x) \mathrm{d} x
$$

The random variable $1 / \Phi_{t}$ can be used to introduce a measure $\mathbb{B}$ on $\left(\Omega, \mathcal{F}_{t}\right)$, which we call the "bridge measure". The option price can then be written:

$$
C_{0}=P_{0 t} \mathbb{E}^{\mathbb{B}}\left[\left(\int_{0}^{\infty}\left(P_{t T} x-K\right) p_{t}(x) \mathrm{d} x\right)^{+}\right]
$$

The special feature of the bridge measure, as we establish in Section XIV in a more general context, is that the random variable $\xi_{t}$ is Gaussian under $\mathbb{B}$. In particular, under $\mathbb{B}$ we find that $\left\{\xi_{t}\right\}$ has mean 0 and variance $t(T-t) / T$. Since $p_{t}(x)$ can be expressed as a function of $\xi_{t}$, when we calculate the expectation in (29) we obtain a tractable formula for $C_{0}$.

To determine the value of the option we define a constant $\xi^{*}$ (the critical value) by the following condition:

$$
\int_{0}^{\infty}\left(P_{t T} x-K\right) p(x) \exp \left[\frac{T}{T-t}\left(\sigma x \xi^{*}-\frac{1}{2} \sigma^{2} x^{2} t\right)\right] \mathrm{d} x=0 .
$$


Then the expectation in (29) can be performed and we find that the option price is

$$
C_{0}=P_{0 T} \int_{0}^{\infty} x p(x) N\left(-z^{*}+\sigma x \sqrt{\tau}\right) \mathrm{d} x-P_{0 t} K \int_{0}^{\infty} p(x) N\left(-z^{*}+\sigma x \sqrt{\tau}\right) \mathrm{d} x,
$$

where $N(x)$ is the standard normal distribution function, and

$$
\tau=\frac{t T}{T-t}, \quad z^{*}=\xi^{*} \sqrt{\frac{T}{t(T-t)}} .
$$

We see that a tractable expression of the Black-Scholes-Merton-Merton type is obtained. The option pricing problem, even for general $p(x)$, reduces to an elementary numerical problem. It is interesting to note that although the probability distribution for the price $S_{t}$ is not of a "standard" type, nevertheless the option valuation problem remains a solvable one.

\section{EXAMPLES OF SPECIFIC DIVIDEND STRUCTURES}

In this section we consider the dynamics of assets with various dividend structures. First we look at a simple asset for which the cash flow is exponentially distributed. The a priori probability density for $D_{T}$ is thus of the form

$$
p(x)=\frac{1}{\delta} \exp (-x / \delta),
$$

where $\delta$ is a constant. We can regard the idea of an exponentially distributed payout as a model for the situation where little is known about the probability distribution of the dividend, apart from its mean. Then from formula (12) we find that the asset price is:

$$
S_{t}=\mathbb{1}_{\{t<T\}} P_{t T} \frac{\int_{0}^{\infty} x \exp (-x / \delta) \exp \left[\frac{T}{T-t}\left(\sigma x \xi_{t}-\frac{1}{2} \sigma^{2} x^{2} t\right)\right] \mathrm{d} x}{\int_{0}^{\infty} \exp (-x / \delta) \exp \left[\frac{T}{T-t}\left(\sigma x \xi_{t}-\frac{1}{2} \sigma^{2} x^{2} t\right)\right] \mathrm{d} x} .
$$

We note that $S_{0}=P_{0 T} \delta$, so we can calibrate $\delta$ by use of the initial price. The integrals in the numerator and denominator in the expression above can be worked out explicitly. Hence, we obtain a closed-form expression for the price in the case of a simple asset with an exponentially-distributed cash flow:

$$
S_{t}=\mathbb{1}_{\{t<T\}} P_{t T}\left[\frac{\exp \left(-\frac{1}{2} B_{t}^{2} / A_{t}\right)}{\sqrt{2 \pi A_{t}} N\left(B_{t} / \sqrt{A_{t}}\right)}+\frac{B_{t}}{A_{t}}\right],
$$

where $A_{t}=\sigma^{2} t T /(T-t)$ and $B_{t}=\sigma T \xi_{t} /(T-t)-\delta^{-1}$.

Next we consider the case of an asset for which the single dividend paid at $T$ is gammadistributed. More specifically, we assume the probability density is of the form

$$
p(x)=\frac{\delta^{n}}{(n-1) !} x^{n-1} \exp (-\delta x),
$$

where $\delta$ is a positive real number and $n$ is a positive integer. This choice for the probability density also leads to a closed-form expression for the share price. We find that

$$
S_{t}=\mathbb{1}_{\{t<T\}} P_{t T} \frac{\sum_{k=0}^{n}\left(\begin{array}{c}
n \\
k
\end{array}\right) A_{t}^{\frac{1}{2} k-n} B_{t}^{n-k} F_{k}\left(-B_{t} / \sqrt{A_{t}}\right)}{\sum_{k=0}^{n-1}\left(\begin{array}{c}
n-1 \\
k
\end{array}\right) A_{t}^{\frac{1}{2} k-n+1} B_{t}^{n-k-1} F_{k}\left(-B_{t} / \sqrt{A_{t}}\right)},
$$


where $A_{t}$ and $B_{t}$ are as above, and

$$
F_{k}(x)=\int_{x}^{\infty} z^{k} \exp \left(-\frac{1}{2} z^{2}\right) d z .
$$

A recursion formula can be worked out for the function $F_{k}(x)$. This is given by

$$
(k+1) F_{k}(x)=F_{k+2}(x)-x^{k+1} \exp \left(-\frac{1}{2} x^{2}\right),
$$

from which it follows that $F_{0}(x)=\sqrt{2 \pi} N(-x), F_{1}(x)=\mathrm{e}^{-\frac{1}{2} x^{2}}, F_{2}(x)=x \mathrm{e}^{-\frac{1}{2} x^{2}}+\sqrt{2 \pi} N(-x)$, $F_{3}(x)=\left(x^{2}+2\right) \mathrm{e}^{-\frac{1}{2} x^{2}}$, and so on. In general, the polynomial parts of $\left\{F_{k}(x)\right\}_{k=0,1,2, \ldots}$ are related to the Legendre polynomials.

\section{MARKET FACTORS AND MULTIPLE CASH FLOWS}

In this section we proceed to consider the more general situation where the asset pays multiple dividends. This will allow us to consider a wider range of financial instruments. Let us write $D_{T_{k}}(k=1, \ldots, n)$ for a set of random dividends paid at the pre-designated dates $T_{k}(k=1, \ldots, n)$. Possession of the asset at time $t$ entitles the bearer to the cash flows occurring at times $T_{k}>t$. For simplicity we assume $n$ is finite. For each value of $k$ we introduce a set of independent random variables $X_{T_{k}}^{\alpha}\left(\alpha=1, \ldots, m_{k}\right)$, which we call market factors or $X$-factors. For each value of $\alpha$ we assume that the market factor $X_{T_{k}}^{\alpha}$ is $\mathcal{F}_{T_{k}}$-measurable, where $\left\{\mathcal{F}_{t}\right\}$ is the market filtration.

For each value of $k$, the market factors $\left\{X_{T_{j}}^{\alpha}\right\}_{j \leq k}$ represent the independent elements that determine the cash flow occurring at time $T_{k}$. Thus for each value of $k$ the cash flow $D_{T_{k}}$ is assumed to have the following structure:

$$
D_{T_{k}}=\Delta_{T_{k}}\left(X_{T_{1}}^{\alpha}, X_{T_{2}}^{\alpha}, \ldots, X_{T_{k}}^{\alpha}\right),
$$

where $\Delta_{T_{k}}\left(X_{T_{1}}^{\alpha}, X_{T_{2}}^{\alpha}, \ldots, X_{T_{k}}^{\alpha}\right)$ is a function of $\sum_{j=1}^{k} m_{j}$ variables. For each cash flow it is, so to speak, the job of the financial analyst (or actuary) to determine the relevant independent market factors, and the form of the cash-flow function $\Delta_{T_{k}}$ for each cash flow. With each market factor $X_{T_{k}}^{\alpha}$ we associate an information process $\left\{\xi_{t T_{k}}^{\alpha}\right\}_{0 \leq t \leq T_{k}}$ of the form

$$
\xi_{t T_{k}}^{\alpha}=\sigma_{T_{k}}^{\alpha} X_{T_{k}}^{\alpha} t+\beta_{t T_{k}}^{\alpha} .
$$

Here $\sigma_{T_{k}}^{\alpha}$ is a parameter, and $\left\{\beta_{t T_{k}}^{\alpha}\right\}$ is a standard Brownian bridge over the interval $\left[0, T_{k}\right]$. We assume that the $X$-factors and the Brownian bridge processes are all independent. The parameter $\sigma_{T_{k}}^{\alpha}$ determines the rate at which the market factor $X_{T_{k}}^{\alpha}$ is revealed. The Brownian bridge represents the associated noise. We assume that the market filtration $\left\{\mathcal{F}_{t}\right\}$ is generated by the totality of the independent information processes $\left\{\xi_{t T_{k}}^{\alpha}\right\}_{0 \leq t \leq T_{k}}$ for $k=1,2, \ldots, n$ and $\alpha=1,2, \ldots, m_{k}$. Hence, the price of the asset is given by

$$
S_{t}=\sum_{k=1}^{n} \mathbb{1}_{\left\{t<T_{k}\right\}} P_{t T_{k}} \mathbb{E}_{t}\left[D_{T_{k}}\right] .
$$




\section{GEOMETRIC BROWNIAN MOTION MODEL}

The simplest application of the $X$-factor technique arises in the case of geometric Brownian motion models. We consider a limited-liability company that makes a single cash distribution $S_{T}$ at time $T$. We assume that $S_{T}$ has a log-normal distribution under $\mathbb{Q}$, and can be written in the form

$$
S_{T}=S_{0} \exp \left(r T+\nu \sqrt{T} X_{T}-\frac{1}{2} \nu^{2} T\right)
$$

where the market factor $X_{T}$ is normally distributed with mean zero and variance one, and $r>0$ and $\nu>0$ are constants. The information process $\left\{\xi_{t}\right\}$ is taken to be of the form

$$
\xi_{t}=\sigma t X_{T}+\beta_{t T}
$$

where the Brownian bridge $\left\{\beta_{t T}\right\}$ is independent of $X_{T}$, and where the information flow rate is of the special form

$$
\sigma=\frac{1}{\sqrt{T}}
$$

By use of the Bayes formula we find that the conditional probability density is of the Gaussian form:

$$
\pi_{t}(x)=\sqrt{\frac{T}{2 \pi(T-t)}} \exp \left(-\frac{1}{2(T-t)}\left(\sqrt{T} x-\xi_{t}\right)^{2}\right),
$$

and has the following dynamics

$$
\mathrm{d} \pi_{t}(x)=\frac{1}{T-t}\left(\sqrt{T} x-\xi_{t}\right) \pi_{t}(x) \mathrm{d} \xi_{t}
$$

A short calculation then shows that the value of the asset at time $t<T$ is given by

$$
\begin{aligned}
S_{t} & =\mathrm{e}^{-r(T-t)} \mathbb{E}_{t}\left[S_{T}\right] \\
& =\mathrm{e}^{-r(T-t)} \int_{-\infty}^{\infty} S_{0} \mathrm{e}^{r T+\nu \sqrt{T} x-\frac{1}{2} \nu^{2} T} \pi_{t}(x) \mathrm{d} x \\
& =S_{0} \exp \left(r t+\nu \xi_{t}-\frac{1}{2} \nu^{2} t\right) .
\end{aligned}
$$

The surprising fact in this example is that $\left\{\xi_{t}\right\}$ itself turns out to be the innovation process. Indeed, it is not too difficult to verify that $\left\{\xi_{t}\right\}$ is an $\left\{\mathcal{F}_{t}\right\}$-Brownian motion. Hence, setting $W_{t}=\xi_{t}$ for $0 \leq t<T$ we obtain the standard geometric Brownian motion model:

$$
S_{t}=S_{0} \exp \left(r t+\nu W_{t}-\frac{1}{2} \nu^{2} t\right) .
$$

We see therefore that starting with an information process of the form (44) we are able to recover the familiar asset price dynamics given by (49).

An important point to note here is that the Brownian bridge process $\left\{\beta_{t T}\right\}$ appears quite naturally in this context. In fact, if we start with (49) then we can make use of the following orthogonal decomposition of the Brownian motion (see, e.g., Yor [24]):

$$
W_{t}=\frac{t}{T} W_{T}+\left(W_{t}-\frac{t}{T} W_{T}\right) .
$$


The second term in the right, independent of the first term on the right, is a standard representation for a Brownian bridge process:

$$
\beta_{t T}=W_{t}-\frac{t}{T} W_{T}
$$

Thus by writing $X_{T}=W_{T} / \sqrt{T}$ and $\sigma=1 / \sqrt{T}$ we find that the right side of (150) is indeed the market information. In other words, formulated in the information-based framework, the standard Black-Scholes-Merton theory can be expressed in terms of a normally distributed $X$-factor and an independent Brownian bridge noise process.

\section{DIVIDEND GROWTH}

As an elementary example of a multi-dividend structure, we shall look at a simple growth model for dividends in the equity markets. We consider an asset that pays a sequence of dividends $D_{T_{k}}$, where each dividend date has an associated $X$-factor. Let $\left\{X_{T_{k}}\right\}_{k=1, \ldots, n}$ be a set of independent, identically-distributed $X$-factors, each with mean $1+g$. The dividend structure is assumed to be of the form

$$
D_{T_{k}}=D_{0} \prod_{j=1}^{k} X_{T_{j}}
$$

where $D_{0}$ is a constant. The parameter $g$ can be interpreted as the dividend growth factor, and $D_{0}$ can be understood as representing the most recent dividend before time zero. For the price of the asset we have:

$$
S_{t}=D_{0} \sum_{k=1}^{n} \mathbb{1}_{\left\{t<T_{k}\right\}} P_{t T_{k}} \mathbb{E}_{t}\left[\prod_{j=1}^{k} X_{T_{j}}\right]
$$

Since the $X$-factors are independent, the conditional expectation of the product appearing in this expression factorises into a product of conditional expectations, and each such conditional expectation can be written in the form of an expression of the type we have already considered. As a consequence we are led to a tractable family of dividend growth models.

\section{ASSETS WITH COMMON FACTORS}

The multiple-dividend asset pricing model introduced in Section VIII can be extended in a very natural way to the situation where two or more assets are being priced. In this case we consider a collection of $N$ assets with price processes $\left\{S_{t}^{(i)}\right\}_{i=1,2, \ldots, N}$. With asset number $(i)$ we associate the cash flows $\left\{D_{T_{k}}^{(i)}\right\}$ paid at the dates $\left\{T_{k}\right\}_{k=1,2, \ldots, n}$. We note that the dates $\left\{T_{k}\right\}_{k=1,2, \ldots, n}$ are not tied to any specific asset, but rather represent the totality of possible cash-flow dates of any of the given assets. If a particular asset has no cash flow on one of the dates, then it is assigned a zero cash-flow for that date. From this point, the theory proceeds exactly as in the single asset case. That is to say, with each value of $k$ we associate a set of $X$-factors $X_{T_{k}}^{\alpha}\left(\alpha=1,2, \ldots, m_{k}\right)$, and a system of market information processes $\left\{\xi_{t T_{k}}^{\alpha}\right\}$. The 
$X$-factors and the information processes are not tied to any particular asset. The cash flow $D_{T_{k}}^{(i)}$ occurring at time $T_{k}$ for asset number $(i)$ is given by a cash flow function of the form

$$
D_{T_{k}}^{(i)}=\Delta_{T_{k}}^{(i)}\left(X_{T_{1}}^{\alpha}, X_{T_{2}}^{\alpha}, \ldots, X_{T_{k}}^{\alpha}\right) .
$$

In other words, for each asset each cash flow can depend on all of the $X$-factors that have been "activated" at that point. Thus for the general multi-asset model we have the following price process system:

$$
S_{t}^{(i)}=\sum_{k=1}^{n} \mathbb{1}_{\left\{t<T_{k}\right\}} P_{t T_{k}} \mathbb{E}_{t}\left[D_{T_{k}}^{(i)}\right]
$$

It is possible in general for two or more assets to "share" an $X$-factor in association with one or more of the cash flows of each of the assets. This in turn implies that the various assets will have at least one Brownian motion in common in the dynamics of their price processes. We thus obtain a natural model for the correlation structures in the prices of these assets. The intuition is that as new information comes in (whether "true" or "bogus") there will be several different assets all affected by the news, and as a consequence there will be a correlated movement in their prices.

\section{ORIGIN OF UNHEDGEABLE STOCHASTIC VOLATILITY}

Based on the general model introduced in the previous sections, we are now in a position to make an observation concerning the nature of stochastic volatility in the equity markets. In particular, we shall show how a natural framework for stochastic volatility arises in the information-based framework. This is achieved without the need for any ad hoc assumptions concerning the dynamics of the stochastic volatility. In fact, a very specific dynamical model for stochastic volatility is obtained - thus leading to a possible means by which the theory proposed here might be tested.

We shall work out the volatility associated with the dynamics of the asset price process $\left\{S_{t}\right\}$ given by (42). The result is given in Proposition 2 below. First, as an example, we consider the dynamics of an asset that pays a single dividend $D_{T}$ at $T$. We assume that the dividend depends on the market factors $\left\{X_{T}^{\alpha}\right\}_{\alpha=1, \ldots, m}$. For $t<T$ we then have:

$$
\begin{aligned}
S_{t} & =P_{t T} \mathbb{E}^{\mathbb{Q}}\left[\Delta_{T}\left(X_{T}^{1}, \ldots, X_{T}^{m}\right) \mid \xi_{t T}^{1}, \ldots, \xi_{t T}^{m}\right] \\
& =P_{t T} \int \cdots \int \Delta_{T}\left(x^{1}, \ldots, x^{m}\right) \pi_{t T}^{1}\left(x_{1}\right) \cdots \pi_{t T}^{m}\left(x_{m}\right) \mathrm{d} x_{1} \cdots \mathrm{d} x_{m} .
\end{aligned}
$$

Here the various conditional probability density functions $\pi_{t T}^{\alpha}(x)$ for $\alpha=1, \ldots, m$ are

$$
\pi_{t T}^{\alpha}(x)=\frac{p^{\alpha}(x) \exp \left[\frac{T}{T-t}\left(\sigma^{\alpha} x \xi_{t T}^{\alpha}-\frac{1}{2}\left(\sigma^{\alpha}\right)^{2} x^{2} t\right)\right]}{\int_{0}^{\infty} p^{\alpha}(x) \exp \left[\frac{T}{T-t}\left(\sigma^{\alpha} x \xi_{t T}^{\alpha}-\frac{1}{2}\left(\sigma^{\alpha}\right)^{2} x^{2} t\right)\right] \mathrm{d} x},
$$

where $p^{\alpha}(x)$ denotes the a priori probability density function for the factor $X_{T}^{\alpha}$. The drift of $\left\{S_{t}\right\}_{0 \leq t<T}$ is given by the short rate. This is because $\mathbb{Q}$ is the risk-neutral measure, and no dividend is paid before $T$. Thus, we are left with the problem of determining the volatility of $\left\{S_{t}\right\}$. We find that for $t<T$ the dynamical equation of $\left\{S_{t}\right\}$ assumes the form:

$$
\mathrm{d} S_{t}=r_{t} S_{t} \mathrm{~d} t+\sum_{\alpha=1}^{m} \Gamma_{t T}^{\alpha} \mathrm{d} W_{t}^{\alpha} .
$$


Here the volatility term associated with factor number $\alpha$ is given by

$$
\Gamma_{t T}^{\alpha}=\sigma^{\alpha} \frac{T}{T-t} P_{t T} \operatorname{Cov}\left[\Delta_{T}\left(X_{T}^{1}, \ldots, X_{T}^{m}\right), X_{T}^{\alpha} \mid \mathcal{F}_{t}\right]
$$

and $\left\{W_{t}^{\alpha}\right\}$ denotes the Brownian motion associated with the information process $\left\{\xi_{t}^{\alpha}\right\}$, as defined in (17). The absolute volatility of $\left\{S_{t}\right\}$ is of the form

$$
\Gamma_{t}=\left(\sum_{\alpha=1}^{m}\left(\Gamma_{t T}^{\alpha}\right)^{2}\right)^{1 / 2} .
$$

For the dynamics of a multi-factor single-dividend paying asset we can thus write $\mathrm{d} S_{t}=$ $r_{t} S_{t} \mathrm{~d} t+\Gamma_{t} \mathrm{~d} Z_{t}$, where the $\left\{\mathcal{F}_{t}\right\}$-Brownian motion $\left\{Z_{t}\right\}$ that drives the asset-price process is

$$
Z_{t}=\int_{0}^{t} \frac{1}{\Gamma_{s}} \sum_{\alpha=1}^{m} \Gamma_{s T}^{\alpha} \mathrm{d} W_{s}^{\alpha} .
$$

The point to note here is that in the case of a multi-factor model we obtain an unhedgeable stochastic volatility. That is to say, although the asset price is in effect driven by a single Brownian motion, its volatility in general depends on a multiplicity of Brownian motions. This means that in general an option position cannot be hedged with a position in the underlying asset. The components of the volatility vector are given by the covariances of the cash flow and the independent market factors. Unhedgeable stochastic volatility thus emerges from the multiplicity of uncertain elements in the market that affect the value of the future cash flow. As a consequence we see that in this framework we obtain a natural explanation for the origin of stochastic volatility.

This result can be contrasted with, say, the Heston model [12], which despite its popularity suffers from the fact that it is ad hoc in nature. Much the same can be said for the various generalisations of the Heston model used in commercial applications. The approach to stochastic volatility proposed in the present paper is thus of a new character. Expression (58) generalises to the case for which the asset pays a set of dividends $D_{T_{k}}(k=1, \ldots, n)$, and for each $k$ the dividend depends on the $X$-factors $\left\{\left\{X_{T_{j}}^{\alpha}\right\}_{j=1, \ldots, k}^{\alpha=1, \ldots, m_{j}}\right\}$. The result can be summarised as follows.

Proposition 2. The price process of a multi-dividend asset has the following dynamics:

$$
\begin{aligned}
\mathrm{d} S_{t}= & r_{t} S_{t} \mathrm{~d} t+\sum_{k=1}^{n} \sum_{\alpha=1}^{m_{k}} \mathbb{1}_{\left\{t<T_{k}\right\}} \frac{\sigma_{k}^{\alpha} T_{k}}{T_{k}-t} P_{t T_{k}} \operatorname{Cov}\left[D_{T_{k}}, X_{T_{k}}^{\alpha} \mid \mathcal{F}_{t}\right] \mathrm{d} W_{t}^{\alpha k} \\
& +\sum_{k=1}^{n} D_{T_{k}} \mathrm{~d} \mathbb{1}_{\left\{t<T_{k}\right\}},
\end{aligned}
$$

where $D_{T_{k}}=\Delta_{T_{k}}\left(X_{T_{1}}^{\alpha}, X_{T_{2}}^{\alpha}, \cdots, X_{T_{k}}^{\alpha}\right)$ is the dividend at time $T_{k}(k=1,2, \ldots, n)$.

\section{TIME-DEPENDENT INFORMATION FLOW}

We consider now a generalisation of the foregoing material to the situation in which the information-flow rate varies in time. The time-dependent problem is of relevance to many 
circumstances. For example, there will typically be more activity in a market during the day than at night - such a consideration is important for short-term investments. Alternatively, it may be that the annual report of a firm is going to be published on a specified day -in this case much more information concerning the future of the firm may be made available on that day than normal.

We begin our analysis of the time-dependent case by considering the situation where there is a single cash flow $D_{T}$ occurring at $T$, and the associated market factor is the cash flow itself. In this way we can focus our attention on mathematical issues arising from the time dependence of the information flow rate. Once these issues have been dealt with, we shall consider more complicated cash-flow structures. For the market information process we propose an expression of the form

$$
\xi_{t}=D_{T} \int_{0}^{t} \sigma_{s} \mathrm{~d} s+\beta_{t T}
$$

where the function $\left\{\sigma_{s}\right\}_{o \leq s \leq T}$ is taken to be deterministic and nonnegative. We assume that $0<\int_{0}^{T} \sigma_{s}^{2} d s<\infty$. The price process $\left\{S_{t}\right\}$ of the asset is then given by

$$
S_{t}=\mathbb{1}_{\{t<T\}} P_{t T} \mathbb{E}\left[D_{T} \mid \mathcal{F}_{t}\right]
$$

where the market filtration is, as in the previous sections, assumed to be generated by the information process.

Our first task is to work out the conditional expectation in (64). This can be achieved by use of a change-of-measure technique, which will be outlined in Section XIV. It will be useful, however, to state the result first. We define the conditional probability density process $\left\{\pi_{t}(x)\right\}$ by setting

$$
\pi_{t}(x)=\frac{\mathrm{d}}{\mathrm{d} x} \mathbb{Q}\left(D_{T} \leq x \mid \mathcal{F}_{t}\right)
$$

The following result is obtained:

Proposition 3. Let the information process $\left\{\xi_{t}\right\}$ be given by (63). Then the conditional probability density process $\left\{\pi_{t}(x)\right\}$ for the random variable $D_{T}$ is given by

$$
\pi_{t}(x)=\frac{p(x) \mathrm{e}^{x\left(\frac{1}{T-t} \xi_{t} \int_{0}^{t} \sigma_{s} \mathrm{~d} s+\int_{0}^{t} \sigma_{s} \mathrm{~d} \xi_{s}\right)-\frac{1}{2} x^{2}\left(\frac{1}{T-t}\left(\int_{0}^{t} \sigma_{s} \mathrm{~d} s\right)^{2}+\int_{0}^{t} \sigma_{s}^{2} \mathrm{~d} s\right)}}{\int_{0}^{\infty} p(x) \mathrm{e}^{x\left(\frac{1}{T-t} \xi_{t} \int_{0}^{t} \sigma_{s} \mathrm{~d} s+\int_{0}^{t} \sigma_{s} \mathrm{~d} \xi_{s}\right)-\frac{1}{2} x^{2}\left(\frac{1}{T-t}\left(\int_{0}^{t} \sigma_{s} \mathrm{~d} s\right)^{2}+\int_{0}^{t} \sigma_{s}^{2} \mathrm{~d} s\right)} \mathrm{d} x} .
$$

We deduce at once from Proposition 3 that the conditional expectation of the random variable $D_{T}$ is

$$
D_{t T}=\frac{\int_{0}^{\infty} x p(x) \mathrm{e}^{x\left(\frac{1}{T-t} \xi_{t} \int_{0}^{t} \sigma_{s} \mathrm{~d} s+\int_{0}^{t} \sigma_{s} \mathrm{~d} \xi_{s}\right)-\frac{1}{2} x^{2}\left(\frac{1}{T-t}\left(\int_{0}^{t} \sigma_{s} \mathrm{~d} s\right)^{2}+\int_{0}^{t} \sigma_{s}^{2} \mathrm{~d} s\right)} \mathrm{d} x}{\int_{0}^{\infty} p(x) \mathrm{e}^{x\left(\frac{1}{T-t} \xi_{t} \int_{0}^{t} \sigma_{s} \mathrm{~d} s+\int_{0}^{t} \sigma_{s} \mathrm{~d} \xi_{s}\right)-\frac{1}{2} x^{2}\left(\frac{1}{T-t}\left(\int_{0}^{t} \sigma_{s} \mathrm{~d} s\right)^{2}+\int_{0}^{t} \sigma_{s}^{2} \mathrm{~d} s\right)} \mathrm{d} x} .
$$

The associated price process $\left\{S_{t}\right\}$ is then given by $S_{t}=\mathbb{1}_{\{t<T\}} P_{t T} D_{t T}$. 


\section{CHANGES OF MEASURE FOR BROWNIAN BRIDGES}

Since the information process is a Brownian bridge with a random drift, we shall require formulae relating a Brownian bridge with drift in one measure to a standard Brownian bridge in another measure to establish Proposition 3 . We proceed as follows. First we recall a wellknown integral representation for the Brownian bridge. Let the probability space $(\Omega, \mathcal{F}, \mathbb{Q})$ be given, with a filtration $\left\{\mathcal{G}_{t}\right\}_{0 \leq t<\infty}$, and let $\left\{B_{t}\right\}$ be a standard $\left\{\mathcal{G}_{t}\right\}$-Brownian motion. Then the process $\left\{\beta_{t T}\right\}$, defined by

$$
\beta_{t T}=(T-t) \int_{0}^{t} \frac{1}{T-s} \mathrm{~d} B_{s},
$$

for $0 \leq t<T$, and by $\beta_{t T}=0$ for $t=T$, is a standard Brownian bridge over the interval $[0, T]$. Expression (68) converges to zero as $t \rightarrow T$; see, e.g., Karatzas and Shreve [16], Protter [21]). The filtration $\left\{\mathcal{G}_{t}\right\}$ is larger than the market filtration $\left\{\mathcal{F}_{t}\right\}$. In particular, since $\left\{\beta_{t T}\right\}$ is adapted to $\left\{\mathcal{G}_{t}\right\}$ we can think of $\left\{\mathcal{G}_{t}\right\}$ as the filtration describing the information available to an "insider" who can distinguish between what is noise and what is not.

Let $D_{T}$ be a random variable on $(\Omega, \mathcal{F}, \mathbb{Q})$. We assume that $D_{T}$ is $\mathcal{G}_{0}$-measurable and that $D_{T}$ is independent of $\left\{\beta_{t T}\right\}$. Thus the value of $D_{T}$ is known "all along" to the insider, but not to the typical market participant. For simplicity in what follows we assume that $D_{T}$ is bounded; this condition can be relaxed with the introduction of an appropriate Novikov-type condition; but we will not pursue the more general situation here. Define the deterministic nonnegative process $\left\{\nu_{t}\right\}_{0 \leq t \leq T}$ by

$$
\nu_{t}=\sigma_{t}+\frac{1}{T-t} \int_{0}^{t} \sigma_{s} \mathrm{~d} s,
$$

and let $\left\{\xi_{t}\right\}$ be defined as in (63). We define the process $\left\{\Lambda_{t}\right\}_{0 \leq t<T}$ by the relation

$$
\frac{1}{\Lambda_{t}}=\exp \left(-D_{T} \int_{0}^{t} \nu_{s} \mathrm{~d} B_{s}-\frac{1}{2} D_{T}^{2} \int_{0}^{t} \nu_{s}^{2} \mathrm{~d} s\right) .
$$

With these elements in hand, we fix a time horizon $U \in(0, T)$ and introduce a probability measure $\mathbb{B}$ on $\mathcal{G}_{U}$ by the relation

$$
\mathrm{d} \mathbb{B}=\Lambda_{U}^{-1} \mathrm{~d} \mathbb{Q} .
$$

Then we have the following facts: (i) The process $\left\{W_{t}^{*}\right\}_{0 \leq t<U}$ defined by

$$
W_{t}^{*}=D_{T} \int_{0}^{t} \nu_{s} \mathrm{~d} s+B_{t}
$$

is a $\mathbb{B}$-Brownian motion. (ii) The process $\left\{\xi_{t}\right\}$ defined by (63) is a $\mathbb{B}$-Brownian bridge and is independent of $D_{T}$. (iii) The random variable $D_{T}$ has the same probability law with respect to $\mathbb{B}$ and $\mathbb{Q}$. (iv) The conditional expectation for any integrable function $f\left(D_{T}\right)$ of the random variable $D_{T}$ can be expressed in the form

$$
\mathbb{E}^{\mathbb{Q}}\left[f\left(D_{T}\right) \mid \mathcal{F}_{t}^{\xi}\right]=\frac{\mathbb{E}^{\mathbb{B}}\left[f\left(D_{T}\right) \Lambda_{t} \mid \mathcal{F}_{t}^{\xi}\right]}{\mathbb{E}^{\mathbb{B}}\left[\Lambda_{t} \mid \mathcal{F}_{t}^{\xi}\right]} .
$$


We note that the measure $\mathbb{B}$ is independent of the specific choice of the time horizon $U$ in the sense that if $\mathbb{B}$ is defined on $\mathcal{G}_{U^{\prime}}$ for some $U^{\prime}>U$, then the restriction of that measure to $\mathcal{G}_{U}$ agrees with the measure $\mathbb{B}$ as already defined.

When we say that $\left\{\xi_{t}\right\}$ is a $\mathbb{B}$-Brownian bridge what we mean, more precisely, is that $\xi_{0}=0$, that $\left\{\xi_{t}\right\}$ is $\mathbb{B}$-Gaussian, that $\mathbb{E}^{\mathbb{B}}\left[\xi_{t}\right]=0$, and that

$$
\mathbb{E}^{\mathbb{B}}\left[\xi_{s} \xi_{t}\right]=\frac{s(T-t)}{T}
$$

for $0 \leq s \leq t \leq U$. Thus with respect to the measure $\mathbb{B}$ the process $\left\{\xi_{t}\right\}_{0 \leq t \leq U}$ has the properties of a standard $[0, T]$-Brownian bridge that has been truncated at time $\bar{U}$. The fact that $\left\{\xi_{t}\right\}$ is a $\mathbb{B}$-Brownian bridge can be verified as follows. By (63), (68), and (172) we have

$$
\begin{aligned}
\xi_{t} & =D_{T} \int_{0}^{t} \sigma_{s} \mathrm{~d} s+(T-t) \int_{0}^{t} \frac{1}{T-s} \mathrm{~d} B_{s} \\
& =D_{T} \int_{0}^{t} \sigma_{s} \mathrm{~d} s+(T-t) \int_{0}^{t} \frac{1}{T-s}\left(\mathrm{~d} W_{s}^{*}-D_{T} \nu_{s} \mathrm{~d} s\right) \\
& =D_{T}\left(\int_{0}^{t} \sigma_{s} \mathrm{~d} s-(T-t) \int_{0}^{t} \frac{1}{T-s} \nu_{s} \mathrm{~d} s\right)+(T-t) \int_{0}^{t} \frac{1}{T-s} \mathrm{~d} W_{s}^{*} \\
& =(T-t) \int_{0}^{t} \frac{1}{T-s} \mathrm{~d} W_{s}^{*},
\end{aligned}
$$

where in the final step we used the relation

$$
\int_{0}^{t} \frac{1}{T-s} \nu_{s} \mathrm{~d} s=\frac{1}{T-t} \int_{0}^{t} \sigma_{s} \mathrm{~d} s .
$$

This formula can be verified explicitly by differentiation, which then gives us (69). In (75) we see that $\left\{\xi_{t}\right\}$ has been given the standard integral representation of a Brownian bridge.

We remark, incidentally, that (73) can be thought of a variation of the Kallianpur-Striebel formula appearing in the literature of nonlinear filtering (see, for example, Bucy and Joseph [4], Davis and Marcus [7], Fujisaki et al. [9], Kallianpur and Striebel [15], Krishnan [17], and Liptser and Shiryaev [18]).

\section{DERIVATION OF THE CONDITIONAL DENSITY}

We have introduced the idea of measure changes associated with Brownian bridges in order to introduce formula (73), which involves the density process $\left\{\Lambda_{t}\right\}$, which in (70) is defined in terms of the $\mathbb{Q}$-Brownian motion $\left\{B_{t}\right\}$. On the other hand, the expectations in (73) are conditional with respect to the information generated by $\left\{\xi_{t}\right\}$. Therefore, it will be convenient to express $\left\{\Lambda_{t}\right\}$ in terms of $\left\{\xi_{t}\right\}$. To do this we substitute (72) in (170) to obtain

$$
\Lambda_{t}=\exp \left(D_{T} \int_{0}^{t} \nu_{s} \mathrm{~d} W_{s}^{*}-\frac{1}{2} D_{T}^{2} \int_{0}^{t} \nu_{s}^{2} \mathrm{~d} s\right) .
$$

We then observe, by differentiating (75), that

$$
\mathrm{d} \xi_{t}=-\frac{\xi_{t}}{T-t} \mathrm{~d} t+\mathrm{d} W_{t}^{*}
$$


Substituting this relation in (77) we obtain

$$
\Lambda_{t}=\exp \left[D_{T}\left(\int_{0}^{t} \nu_{s} \mathrm{~d} \xi_{s}+\int_{0}^{t} \frac{1}{T-s} \nu_{s} \xi_{s} \mathrm{~d} s\right)-\frac{1}{2} D_{T}^{2} \int_{0}^{t} \nu_{s}^{2} \mathrm{~d} s\right] .
$$

In principle at this point all we need to do is to substitute (69) into (79) to obtain the result for $\left\{\Lambda_{t}\right\}$. In practice, further simplification can be achieved. To this end, we note that by taking the differential of the coefficient of $D_{T}$ in the exponent of (79) we get

$$
\begin{aligned}
\mathrm{d}\left(\int_{0}^{t} \nu_{s} \mathrm{~d} \xi_{s}+\int_{0}^{t} \frac{1}{T-s} \nu_{s} \xi_{s} \mathrm{~d} s\right) & =\nu_{t}\left(\mathrm{~d} \xi_{t}+\frac{1}{T-t} \xi_{t} \mathrm{~d} t\right) \\
& =\left(\sigma_{t}+\frac{1}{T-t} \int_{0}^{t} \sigma_{s} \mathrm{~d} s\right)\left(\mathrm{d} \xi_{t}+\frac{1}{T-t} \xi_{t} \mathrm{~d} t\right) \\
& =\mathrm{d}\left(\frac{1}{T-t} \xi_{t} \int_{0}^{t} \sigma_{s} \mathrm{~d} s+\int_{0}^{t} \sigma_{s} \mathrm{~d} \xi_{s}\right) .
\end{aligned}
$$

Then integrating both sides of (80) we obtain:

$$
\int_{0}^{t} \nu_{s} \mathrm{~d} \xi_{s}+\int_{0}^{t} \frac{1}{T-s} \nu_{s} \xi_{s} \mathrm{~d} s=\frac{1}{T-t} \xi_{t} \int_{0}^{t} \sigma_{s} \mathrm{~d} s+\int_{0}^{t} \sigma_{s} \mathrm{~d} \xi_{s} .
$$

Similarly, by taking the differential of the coefficient of $-\frac{1}{2} D_{T}^{2}$ in the exponent of (799) and making use of (69), we find

$$
\begin{aligned}
\nu_{t}^{2} \mathrm{~d} t & =\left[\sigma_{t}^{2}+2 \frac{1}{T-t} \sigma_{t} \int_{0}^{t} \sigma_{s} \mathrm{~d} s+\frac{1}{(T-t)^{2}}\left(\int_{0}^{t} \sigma_{s} \mathrm{~d} s\right)^{2}\right] \mathrm{d} t \\
& =\mathrm{d}\left[\frac{1}{T-t}\left(\int_{0}^{t} \sigma_{s} \mathrm{~d} s\right)^{2}+\int_{0}^{t} \sigma_{s}^{2} \mathrm{~d} s\right] .
\end{aligned}
$$

Therefore, by integrating both sides of (82) we obtain an identity for the coefficient of $-\frac{1}{2} D_{T}^{2}$.

It follows by virtue of the two identities just obtained that $\left\{\Lambda_{t}\right\}$ can be expressed in terms of $\left\{\xi_{t}\right\}$. More explicitly, we have

$$
\Lambda_{t}=\exp \left[D_{T}\left(\frac{1}{T-t} \xi_{t} \int_{0}^{t} \sigma_{s} \mathrm{~d} s+\int_{0}^{t} \sigma_{s} \mathrm{~d} \xi_{s}\right)-\frac{1}{2} D_{T}^{2}\left(\frac{1}{T-t}\left(\int_{0}^{t} \sigma_{s} \mathrm{~d} s\right)^{2}+\int_{0}^{t} \sigma_{s}^{2} \mathrm{~d} s\right)\right] .
$$

Note that by transforming (79) into (83) we have eliminated a term having $\left\{\xi_{t}\right\}$ in the integrand, thus achieving a considerable simplification. Proposition 3 can then be deduced if we use equation (76) and the basic relation

$$
\mathbb{Q}\left(D_{T} \leq x \mid \mathcal{F}_{t}\right)=\mathbb{E}^{\mathbb{Q}}\left[\mathbb{1}_{\left\{D_{T} \leq x\right\}} \mid \mathcal{F}_{t}\right] .
$$

In particular, since $D_{T}$ and $\left\{\xi_{t}\right\}$ are independent under the bridge measure, by virtue of (73), (83), and (84) we obtain

$$
\mathbb{Q}\left(D_{T} \leq x \mid \mathcal{F}_{t}\right)=\frac{\int_{0}^{x} p(y) \mathrm{e}^{y\left(\frac{1}{T-t} \xi_{t} \int_{0}^{t} \sigma_{s} \mathrm{~d} s+\int_{0}^{t} \sigma_{s} \mathrm{~d} \xi_{s}\right)-\frac{1}{2} y^{2}\left(\frac{1}{T-t}\left(\int_{0}^{t} \sigma_{s} \mathrm{~d} s\right)^{2}+\int_{0}^{t} \sigma_{s}^{2} \mathrm{~d} s\right)} \mathrm{d} y}{\int_{0}^{\infty} p(y) \mathrm{e}^{y\left(\frac{1}{T-t} \xi_{t} \int_{0}^{t} \sigma_{s} \mathrm{~d} s+\int_{0}^{t} \sigma_{s} \mathrm{~d} \xi_{s}\right)-\frac{1}{2} y^{2}\left(\frac{1}{T-t}\left(\int_{0}^{t} \sigma_{s} \mathrm{~d} s\right)^{2}+\int_{0}^{t} \sigma_{s}^{2} \mathrm{~d} s\right)} \mathrm{d} y}
$$


from which we immediately infer Proposition 3 by differentiation with respect to $x$.

We conclude this section by noting that an alternative expression for $\left\{\pi_{t}(x)\right\}$, written in terms of $\left\{W_{t}^{*}\right\}$, is given by

$$
\pi_{t}(x)=\frac{p(x) \exp \left(x \int_{0}^{t} \nu_{u} \mathrm{~d} W_{u}^{*}-\frac{1}{2} x^{2} \int_{0}^{t} \nu_{u}^{2} \mathrm{~d} u\right)}{\int_{0}^{\infty} p(x) \exp \left(x \int_{0}^{t} \nu_{u} \mathrm{~d} W_{u}^{*}-\frac{1}{2} x^{2} \int_{0}^{t} \nu_{u}^{2} \mathrm{~d} u\right) \mathrm{d} x} .
$$

\section{CONSISTENCY RELATIONS}

Before we proceed to analyse in detail the dynamics of the price process $\left\{S_{t}\right\}$, first we shall establish a useful dynamical consistency relation satisfied by prices obtained in the information-based framework. By "consistency" we have in mind the following. Suppose that we re-initialise the information process at an intermediate time $s \in(0, T)$ by specifying the value $\xi_{s}$ of the information at that time. For the framework to be dynamically consistent, we require that the remainder of the period $[s, T]$ admits a representation in terms of a suitably "renormalised" information process. Specifically, we have:

Proposition 4. Let $0 \leq s \leq t \leq T$. The conditional probability $\pi_{t}(x)$ can be written in terms of the intermediate conditional probability $\pi_{s}(x)$ in the form

$$
\pi_{t}(x)=\frac{\pi_{s}(x) \mathrm{e}^{x\left(\frac{1}{T-t} \eta_{t} \int_{s}^{t} \tilde{\sigma}_{u} \mathrm{~d} u+\int_{s}^{t} \tilde{\sigma}_{u} \mathrm{~d} \eta_{u}\right)-\frac{1}{2} x^{2}\left(\frac{1}{T-t}\left(\int_{s}^{t} \tilde{\sigma}_{u} \mathrm{~d} u\right)^{2}+\int_{s}^{t} \tilde{\sigma}_{u}^{2} \mathrm{~d} u\right)}}{\int_{0}^{\infty} \pi_{s}(x) \mathrm{e}^{x\left(\frac{1}{T-t} \eta_{t} \int_{s}^{t} \tilde{\sigma}_{u} \mathrm{~d} u+\int_{s}^{t} \tilde{\sigma}_{u} \mathrm{~d} \eta_{u}\right)-\frac{1}{2} x^{2}\left(\frac{1}{T-t}\left(\int_{s}^{t} \tilde{\sigma}_{u} \mathrm{~d} u\right)^{2}+\int_{s}^{t} \tilde{\sigma}_{u}^{2} \mathrm{~d} u\right)} \mathrm{d} x},
$$

where

$$
\tilde{\sigma}_{u}=\sigma_{u}+\frac{1}{T-s} \int_{0}^{s} \sigma_{v} \mathrm{~d} v
$$

is the re-initialised market information-flow rate, and

$$
\eta_{t}=\xi_{t}-\frac{T-t}{T-s} \xi_{s}
$$

is the re-initialised information process.

The fact that $\left\{\eta_{t}\right\}_{s \leq t \leq T}$ represents the updated information process bridging the interval $[s, T]$ can be seen as follows. First we note that $\eta_{s}=0$ and that $\eta_{T}=\xi_{T}$. Substituting (63) in (89) we find that

$$
\eta_{t}=D_{T} \int_{s}^{t} \tilde{\sigma}_{u} \mathrm{~d} u+\gamma_{t T}
$$

where $\tilde{\sigma}_{u}$ is as defined in (88), and

$$
\gamma_{t T}=\beta_{t T}-\frac{T-t}{T-s} \beta_{s T}
$$

A calculation making use of the covariance of the Brownian bridge $\left\{\beta_{t T}\right\}$ shows that the Gaussian process $\left\{\gamma_{t T}\right\}_{s \leq t \leq T}$ is a standard Brownian bridge over the interval $[s, T]$. It thus follows that $\left\{\eta_{t}\right\}$ is the information bridge interpolating the interval $[s, T]$. 
To verify (87) we note that (86) can be written in the form

$$
\pi_{t}(x)=\frac{\pi_{s}(x) \exp \left(x \int_{s}^{t} \nu_{u} \mathrm{~d} W_{u}^{*}-\frac{1}{2} x^{2} \int_{s}^{t} \nu_{u}^{2} \mathrm{~d} u\right)}{\int_{0}^{\infty} \pi_{s}(x) \exp \left(x \int_{s}^{t} \nu_{u} \mathrm{~d} W_{u}^{*}-\frac{1}{2} x^{2} \int_{s}^{t} \nu_{u}^{2} \mathrm{~d} u\right) \mathrm{d} x} .
$$

The identity (80) then implies that

$$
\begin{aligned}
\int_{s}^{t} \nu_{u} \mathrm{~d} W_{u}^{*} & =\frac{1}{T-t} \xi_{t} \int_{s}^{t} \sigma_{u} \mathrm{~d} u+\int_{s}^{t} \sigma_{u} \mathrm{~d} \xi_{u}+\left(\frac{\xi_{t}}{T-t}-\frac{\xi_{s}}{T-s}\right) \int_{0}^{s} \sigma_{u} \mathrm{~d} u \\
& =\frac{1}{T-t} \eta_{t} \int_{s}^{t} \tilde{\sigma}_{u} \mathrm{~d} u+\int_{s}^{t} \tilde{\sigma}_{u} \mathrm{~d} \eta_{u}
\end{aligned}
$$

where we have made use of (88) and (89). Similarly, (81) implies that

$$
\begin{aligned}
\int_{s}^{t} \nu_{u}^{2} \mathrm{~d} u & =\frac{1}{T-t}\left(\int_{0}^{t} \sigma_{u} \mathrm{~d} u\right)^{2}-\frac{1}{T-s}\left(\int_{0}^{s} \sigma_{u} \mathrm{~d} u\right)^{2}+\int_{s}^{t} \sigma_{u}^{2} \mathrm{~d} u \\
& =\frac{1}{T-t}\left(\int_{s}^{t} \tilde{\sigma}_{u} \mathrm{~d} u\right)^{2}+\int_{s}^{t} \tilde{\sigma}_{u}^{2} \mathrm{~d} u
\end{aligned}
$$

Substitution of (93) and (94) into (92) establishes (87). In particular, the form of (87) is identical to the original formula (66), modulo the indicated renormalisation of the information process and the associated information flow rate.

\section{EXPECTED DIVIDEND}

The goal of sections XIII, XIV, and XV was to obtain an expression for the conditional expectation (13) in the case of a single-dividend asset in the case of a time-dependent information-flow rate. In the analysis of the associated price process it will therefore be useful to work out the dynamics of the conditional expectation of the dividend. In particular, an application of Ito's rule to (67), after some rearrangement, shows that

$$
\mathrm{d} D_{t T}=\nu_{t} V_{t}\left(\frac{1}{T-t} \xi_{t}-\nu_{t} D_{t T}\right) \mathrm{d} t+\nu_{t} V_{t} \mathrm{~d} \xi_{t}
$$

where $\left\{V_{t}\right\}$ is the conditional variance of the random variable $D_{T}$, given by (16). Let us define a new process $\left\{W_{t}\right\}$ by

$$
W_{t}=\xi_{t}+\int_{0}^{t} \frac{1}{T-s} \xi_{s} \mathrm{~d} s-\int_{0}^{t} \nu_{s} D_{s T} \mathrm{~d} s .
$$

We refer to $\left\{W_{t}\right\}$ as the "innovation process". It follows from the definition of $\left\{W_{t}\right\}$ that

$$
\mathrm{d} D_{t T}=\nu_{t} V_{t} \mathrm{~d} W_{t} .
$$

Since $\left\{D_{t T}\right\}$ is an $\left\{\mathcal{F}_{t}\right\}$-martingale we are thus led to conjecture that $\left\{W_{t}\right\}$ must also be an $\left\{\mathcal{F}_{t}\right\}$-martingale. In fact, we have the following result: 
Proposition 5. The process $\left\{W_{t}\right\}$ defined by $(\underline{96})$ is an $\left\{\mathcal{F}_{t}\right\}$-Brownian motion under $\mathbb{Q}$.

Proof. We need to establish that (i) $\left\{W_{t}\right\}$ is an $\left\{\mathcal{F}_{t}\right\}$-martingale, and that (ii) $\left(\mathrm{d} W_{t}\right)^{2}=$ $\mathrm{d} t$. Writing $\mathbb{E}_{t}[-]=\mathbb{E}^{\mathbb{Q}}\left[-\mid \mathcal{F}_{t}\right]$ and letting $t \leq u$ we have

$$
\mathbb{E}_{t}\left[W_{u}\right]=\mathbb{E}_{t}\left[\xi_{u}\right]+\mathbb{E}_{t}\left[\int_{0}^{u} \frac{1}{T-s} \xi_{s} \mathrm{~d} s\right]-\mathbb{E}_{t}\left[\int_{0}^{u} \nu_{s} D_{s T} \mathrm{~d} s\right] .
$$

Splitting the second two terms on the right into integrals between 0 and $t$, and between $t$ and $u$, we obtain

$$
\begin{aligned}
\mathbb{E}_{t}\left[W_{u}\right]= & \mathbb{E}_{t}\left[\xi_{u}\right]+\int_{0}^{t} \frac{1}{T-s} \xi_{s} \mathrm{~d} s-\int_{0}^{t} \nu_{s} D_{s T} \mathrm{~d} s \\
& +\int_{t}^{u} \frac{1}{T-s} \mathbb{E}_{t}\left[\xi_{s}\right] \mathrm{d} s-\int_{t}^{u} \nu_{s} \mathbb{E}_{t}\left[D_{s T}\right] \mathrm{d} s .
\end{aligned}
$$

The martingale property of the conditional expectation implies that $\mathbb{E}_{t}\left[D_{s T}\right]=D_{t T}$ for $t \leq s$, which allows us to simplify the last term. To simplify the expression for the expectation $\mathbb{E}_{t}\left[\xi_{s}\right]$ for $t \leq s$ we use the tower property:

$$
\mathbb{E}_{t}\left[\beta_{s T}\right]=\mathbb{E}_{t}\left[\mathbb{E}\left[\beta_{s T} \mid H_{T}, \beta_{t T}\right]\right]=\mathbb{E}_{t}\left[\mathbb{E}\left[\beta_{s T} \mid \beta_{t T}\right]\right] .
$$

To calculate the inner expectation $\mathbb{E}\left[\beta_{s T} \mid \beta_{t T}\right]$ here we use the fact that the random variable $\beta_{s T} /(T-s)-\beta_{t T} /(T-t)$ is independent of $\beta_{t T}$ and deduce that

$$
\mathbb{E}\left[\beta_{s T} \mid \beta_{t T}\right]=\frac{T-s}{T-t} \beta_{t T}
$$

from which it follows that

$$
\mathbb{E}_{t}\left[\beta_{s T}\right]=\frac{T-s}{T-t} \mathbb{E}_{t}\left[\beta_{t T}\right]
$$

As a result we obtain

$$
\mathbb{E}_{t}\left[\xi_{s}\right]=D_{t T} \int_{0}^{s} \sigma_{v} \mathrm{~d} v+\frac{T-s}{T-t} \mathbb{E}_{t}\left[\beta_{t T}\right] .
$$

We also recall the definition of $\left\{W_{t}\right\}$ given by (96), which implies that

$$
\int_{0}^{t} \frac{1}{T-s} \xi_{s} \mathrm{~d} s-\int_{0}^{t} \nu_{s} D_{s T} \mathrm{~d} s=W_{t}-\xi_{t} .
$$

Therefore, substituting (103) and (104) into (99) we obtain

$$
\begin{aligned}
\mathbb{E}_{t}\left[W_{u}\right]= & D_{t T} \int_{0}^{u} \sigma_{s} \mathrm{~d} s+W_{t}-\xi_{t}+D_{t T} \int_{t}^{u} \frac{1}{T-s}\left(\int_{0}^{s} \sigma_{v} \mathrm{~d} v\right) \mathrm{d} s-D_{t T} \int_{t}^{u} \nu_{s} \mathrm{~d} s \\
& +\mathbb{E}_{t}\left[\beta_{t T}\right] .
\end{aligned}
$$

Next we split the first term into an integral from 0 to $t$ and an integral from $t$ to $u$, and we insert the definition (69) of $\left\{\nu_{t}\right\}$ into the fifth term. The result is:

$$
\mathbb{E}_{t}\left[W_{u}\right]=W_{t}+D_{t T} \int_{0}^{t} \sigma_{s} \mathrm{~d} s+\mathbb{E}_{t}\left[\beta_{t T}\right]-\xi_{t}
$$


Finally, if we make use of the fact that $\xi_{t}=\mathbb{E}_{t}\left[\xi_{t}\right]$, and hence that

$$
\xi_{t}=D_{t T} \int_{0}^{t} \sigma_{s} \mathrm{~d} s+\mathbb{E}_{t}\left[\beta_{t T}\right]
$$

we see that $\left\{W_{t}\right\}$ satisfies the martingale condition. On the other hand, by virtue of (96) we have $\left(\mathrm{d} W_{t}\right)^{2}=\mathrm{d} t$. We conclude that $\left\{W_{t}\right\}$ is an $\left\{\mathcal{F}_{t}\right\}$-Brownian motion under $\mathbb{Q}$.

\section{ASSET PRICES AND DERIVATIVE PRICES}

We are now in a position to consider in more detail the dynamics of the price process of an asset paying a single dividend $D_{T}$ in the case of a time-dependent information flow. For $\left\{S_{t}\right\}$ we have $S_{t}=\mathbb{1}_{\{t<T\}} P_{t T} D_{t T}$, or equivalently

$$
S_{t}=\mathbb{1}_{\{t<T\}} P_{t T} \frac{\int_{0}^{\infty} x p(x) \mathrm{e}^{x\left(\frac{1}{T-t} \xi_{t} \int_{0}^{t} \sigma_{s} \mathrm{~d} s+\int_{0}^{t} \sigma_{s} \mathrm{~d} \xi_{s}\right)-\frac{1}{2} x^{2}\left(\frac{1}{T-t}\left(\int_{0}^{t} \sigma_{s} \mathrm{~d} s\right)^{2}+\int_{0}^{t} \sigma_{s}^{2} \mathrm{~d} s\right)} \mathrm{d} x}{\int_{0}^{\infty} p(x) \mathrm{e}^{x\left(\frac{1}{T-t} \xi_{t} \int_{0}^{t} \sigma_{s} \mathrm{~d} s+\int_{0}^{t} \sigma_{s} \mathrm{~d} \xi_{s}\right)-\frac{1}{2} x^{2}\left(\frac{1}{T-t}\left(\int_{0}^{t} \sigma_{s} \mathrm{~d} s\right)^{2}+\int_{0}^{t} \sigma_{s}^{2} \mathrm{~d} s\right)} \mathrm{d} x}
$$

A calculation making use of (97) shows that for the dynamics of the price we have

$$
\mathrm{d} S_{t}=r_{t} S_{t} \mathrm{~d} t+\Gamma_{t T} \mathrm{~d} W_{t}
$$

where the asset price volatility is given by $\Gamma_{t T}=\nu_{t} P_{t T} V_{t}$, where $V_{t}$ is the conditional variance of the dividend, given by (16). It should be evident by virtue of its definition that $\left\{V_{t}\right\}$ is a supermartingale. More specifically, for the dynamics of $\left\{V_{t}\right\}$ we obtain

$$
\mathrm{d} V_{t}=-\nu_{t}^{2} V_{t}^{2} \mathrm{~d} t+\nu_{t} \kappa_{t} \mathrm{~d} W_{t},
$$

where $\kappa_{t}$ denotes the third conditional moment of $D_{T}$, given by $\kappa_{t}=\mathbb{E}_{t}\left[\left(D_{T}-D_{t T}\right)^{3}\right]$.

Although we have derived (108) by assuming that the price process is induced by the market information $\left\{\xi_{t}\right\}$, the result to be shown in Section XIX demonstrates that we can regard the dynamical equation (109) for the price process as given, and then deduce the structure of the underlying information. The information-based interpretation of the modelling framework, however, is more appealing. According to this interpretation there is a flow of market information, which is available to all market participants and is represented by the filtration generated by $\left\{\xi_{t}\right\}$. Given this information, each participant will "act", in our interpretation, so as to minimise the risk adjusted future $\mathrm{P} \& \mathrm{~L}$ variance associated with the cash flow under consideration. The future P\&L is determined by the value of $D_{T}$, and the estimate of $D_{T}$ that minimises its variance is indeed given by the conditional expectation (13). By discounting this expectation with $P_{t T}$ we recover the price process $\left\{S_{t}\right\}$.

We note that $\left\{\Gamma_{t T}\right\}$ is "infinitely stochastic" in the sense that all of the higher-order volatilities (the volatility of the volatility, and so on) are stochastic. These higher-order volatilities have a natural interpretation: the volatility of the asset price is determined by the variance of the random cash flow; the volatility of the volatility is determined by the skewness of $D_{T}$; its volatility is determined by the kurtosis of $D_{T}$; and so on.

The fact that the asset price in the bridge measure is given by a function of a Gaussian random variable means that the pricing of derivatives is numerically straightforward. We have seen this in the case of a constant information-flow rate, but the result holds in the 
time-dependent case as well. For example, consider a European-style call option with strike $K$ and maturity $t$, where $t \leq T$, for which the value is (23). If we express the asset price $S_{t}$ on the option maturity date in terms of the $\mathbb{B}$-Brownian motion $\left\{W_{t}^{*}\right\}$ we find

$$
C_{0}=P_{0 t} \mathbb{E}^{\mathbb{Q}}\left[\frac{1}{\Phi_{t}}\left\{\int_{0}^{\infty}\left(P_{t T} x-K\right) p(x) \exp \left(x \int_{0}^{t} \nu_{s} \mathrm{~d} W_{s}^{*}-\frac{1}{2} x^{2} \int_{0}^{t} \nu_{s}^{2} \mathrm{~d} s\right) \mathrm{d} x\right\}^{+}\right],
$$

where

$$
\Phi_{t}=\int_{0}^{\infty} p(x) \exp \left(x \int_{0}^{t} \nu_{s} \mathrm{~d} W_{s}^{*}-\frac{1}{2} x^{2} \int_{0}^{t} \nu_{s}^{2} \mathrm{~d} s\right) \mathrm{d} x
$$

To proceed we shall use the factor $1 / \Phi_{t}$ in (111) to make a change of measure on $\left(\Omega, \mathcal{F}_{t}\right)$. The idea is as follows. We fix a time horizon $u$ at or beyond the option expiration but before the bond maturity, so $t \leq u<T$, and define a process $\left\{\Phi_{t}\right\}_{0 \leq t \leq u}$ by use of the expression (112), where now we let $t$ vary in the range $[0, u]$. By an application of Ito calculus on (112) we see that $\mathrm{d} \Phi_{t}=\nu_{t} D_{t T} \Phi_{t} \mathrm{~d} W_{t}^{*}$. On the other hand, it follows from (78) and (96) that the $\mathbb{B}$-Brownian motion $\left\{W_{t}^{*}\right\}$ and the $\mathbb{Q}$-Brownian motion $\left\{W_{t}\right\}$ are related by

$$
\mathrm{d} W_{t}^{*}=\mathrm{d} W_{t}+\nu_{t} D_{t T} \mathrm{~d} t
$$

Therefore, in terms of $\left\{W_{t}\right\}$ we have

$$
\mathrm{d} \Phi_{t}=\nu_{t}^{2} D_{t T}^{2} \Phi_{t} \mathrm{~d} t+\nu_{t} D_{t T} \Phi_{t} \mathrm{~d} W_{t}
$$

from which it follows that

$$
\mathrm{d} \Phi_{t}^{-1}=-\nu_{t} D_{t T} \Phi_{t}^{-1} \mathrm{~d} W_{t}
$$

Upon integration we deduce

$$
\Phi_{t}^{-1}=\exp \left(-\int_{0}^{t} \nu_{s} D_{s T} \mathrm{~d} W_{s}-\frac{1}{2} \int_{0}^{t} \nu_{s}^{2} D_{s T}^{2} \mathrm{~d} s\right) .
$$

Since $\left\{\nu_{s} D_{s T}\right\}$ is bounded, and $s \leq u<T$, we see that $\left\{\Phi_{s}^{-1}\right\}_{0 \leq s \leq u}$ is a $\mathbb{Q}$-martingale with $\mathbb{E}^{\mathbb{Q}}\left[\Phi_{t}^{-1}\right]=1$, where $t$ is the option maturity date. Therefore, the factor $1 / \Phi_{t}$ in (111) can be used to effect a change of measure $\mathbb{Q} \rightarrow \mathbb{B}$ on $\left(\Omega, \mathcal{F}_{t}\right)$. We note that while on the space $\left(\Omega, \mathcal{G}_{t}\right)$ it is the process $\left\{\Lambda_{t}\right\}$ introduced in (70) that defines the measure change from $\mathbb{B}$ and $\mathbb{Q}$, on $\left(\Omega, \mathcal{F}_{t}\right)$ it is $\Phi_{t}=\mathbb{E}^{\mathbb{Q}}\left[\Lambda_{t} \mid \mathcal{F}_{t}\right]$ that defines the relevant measure change. As a consequence, by changing the measure in (111) we obtain

$$
C_{0}=P_{0 t} \mathbb{E}^{\mathbb{B}}\left[\left\{\int_{0}^{\infty}\left(P_{t T} x-K\right) p(x) \exp \left(x \int_{0}^{t} \nu_{s} \mathrm{~d} W_{s}^{*}-\frac{1}{2} x^{2} \int_{0}^{t} \nu_{s}^{2} \mathrm{~d} s\right) \mathrm{d} x\right\}^{+}\right] .
$$

This result should be compared with (27). We note that in the bridge measure the expression $\int_{0}^{t} \nu_{s} \mathrm{~d} W_{s}^{*}$ is a Gaussian random variable with mean zero and variance

$$
\omega_{t}^{2}=\int_{0}^{t} \nu_{s}^{2} \mathrm{~d} s=\frac{1}{T-t}\left(\int_{0}^{t} \sigma_{s} \mathrm{~d} s\right)^{2}+\int_{0}^{t} \sigma_{s}^{2} \mathrm{~d} s
$$


Here we have used the relation (82). Therefore, if we set

$$
Y=\omega_{t}^{-1} \int_{0}^{t} \nu_{s} \mathrm{~d} W_{s}^{*}
$$

it follows that $Y$ is $\mathbb{B}$-Gaussian. For the call price we thus have

$$
C_{0}=P_{0 t} \mathbb{E}^{\mathbb{B}}\left[\left\{\int_{0}^{\infty}\left(P_{t T} x-K\right) p(x) \mathrm{e}^{\omega_{t} x Y-\frac{1}{2} \omega_{t}^{2} x^{2}} \mathrm{~d} x\right\}^{+}\right]
$$

and hence

$$
C_{0}=P_{0 t} \frac{1}{\sqrt{2 \pi}} \int_{y=-\infty}^{\infty} \mathrm{e}^{-\frac{1}{2} y^{2}}\left(\int_{x=0}^{\infty}\left(P_{t T} x-K\right) p(x) \mathrm{e}^{\omega_{t} x y-\frac{1}{2} \omega_{t}^{2} x^{2}} \mathrm{~d} x\right)^{+} \mathrm{d} y .
$$

We observe that there exists a critical value $y=y^{*}$ such that the argument of the "plus" function vanishes in the expression above. Thus $y^{*}$ is given by

$$
\int_{0}^{\infty}\left(P_{t T} x-K\right) p(x) \mathrm{e}^{\omega_{t} x y^{*}-\frac{1}{2} \omega_{t}^{2} x^{2}} \mathrm{~d} x=0 .
$$

As a consequence the call price can be written

$$
C_{0}=P_{0 t} \frac{1}{\sqrt{2 \pi}} \int_{y=y^{*}}^{\infty} \mathrm{e}^{-\frac{1}{2} y^{2}}\left(\int_{x=0}^{\infty}\left(P_{t T} x-K\right) p(x) \mathrm{e}^{\omega_{t} x y-\frac{1}{2} \omega_{t}^{2} x^{2}} \mathrm{~d} x\right) \mathrm{d} y .
$$

The integration in the $y$ variable can be performed, and we deduce the following representation for the call price:

$$
C_{0}=P_{0 t} \int_{0}^{\infty}\left(P_{t T} x-K\right) p(x) N\left(\omega_{t} x-y^{*}\right) \mathrm{d} x .
$$

When the cash flow is represented by a discrete random variable and the information-flow rate is constant, this result reduces to an expression equivalent to the option pricing formula derived in Brody et al. [3]. If the cash flow is a continuous random variable and the information flow rate is constant then we recover the expression (31) given in section IV (see also Rutkowski and $\mathrm{Yu}[22]$ ).

We conclude this section with the remark that the simulation of $\left\{S_{t}\right\}$ is straightforward. First, we generate a Brownian trajectory, and form the associated Brownian bridge $\left\{\beta_{t T}(\omega)\right\}$. We then select a value for $D_{T}$ by a method consistent with the a priori probability density $p(x)$, and substitute these in the formula $\xi_{t}(\omega)=D_{T}(\omega) \int_{0}^{t} \sigma_{s} \mathrm{~d} s+\beta_{t T}(\omega)$ for some choice of $\left\{\sigma_{t}\right\}$. Finally, substitution of $\left\{\xi_{t}(\omega)\right\}$ in (108) gives us a simulated path $\left\{S_{t}(\omega)\right\}$. The statistics of the process $\left\{S_{t}\right\}$ are obtained by repeating this procedure, the results of which can be used to price derivatives, or to calibrate the information-flow rate $\left\{\sigma_{t}\right\}$.

\section{EXISTENCE OF THE INFORMATION PROCESS}

We consider now what might be called the "inverse problem" for information-based asset pricing. The idea is to begin with the conditional density process $\left\{\pi_{t}(x)\right\}$ and to construct 
from it the independent degrees of freedom represented by the $X$-factor $D_{T}$ and the noise $\left\{\beta_{t T}\right\}$. The setup is as follows. On the probability space $(\Omega, \mathcal{F}, \mathbb{Q})$ let $\left\{W_{t}\right\}$ be a Brownian motion and let $\left\{\mathcal{F}_{t}\right\}$ be the filtration generated by $\left\{W_{t}\right\}$. Let $D_{T}$ be $\mathcal{F}_{T}$-measurable, and let $\left\{\pi_{t}(x)\right\}$ denote the associated conditional probability density process. We assume that $\left\{\pi_{t}(x)\right\}$ satisfies the stochastic differential equation

$$
\mathrm{d} \pi_{t}(x)=\nu_{t}\left(x-D_{t T}\right) \pi_{t}(x) \mathrm{d} W_{t},
$$

with the initial condition $\pi_{0}(x)=p(x)$, where $\left\{\nu_{t}\right\}$ is given by (69), and where

$$
D_{t T}=\int_{0}^{\infty} x \pi_{t}(x) \mathrm{d} x .
$$

We define the process $\left\{\xi_{t}\right\}$ as follows:

$$
\xi_{t}=(T-t) \int_{0}^{t} \frac{1}{T-s}\left(\mathrm{~d} W_{s}+\nu_{s} D_{s T} \mathrm{~d} s\right) .
$$

Then we have the following result:

Proposition 6. The random variables $D_{T}$ and $\beta_{t T}=\xi_{t}-D_{T} \int_{0}^{t} \sigma_{s} \mathrm{~d}$ s are $\mathbb{Q}$-independent for all $t \in[0, T]$. Furthermore, the process $\left\{\beta_{t T}\right\}$ is a $\mathbb{Q}$-Brownian bridge.

Proof. To establish the independence of $D_{T}$ and $\beta_{t T}$ it suffices to verify that

$$
\mathbb{E}^{\mathbb{Q}}\left[\mathrm{e}^{x \beta_{t T}+y D_{T}}\right]=\mathbb{E}^{\mathbb{Q}}\left[\mathrm{e}^{x \beta_{t T}}\right] \mathbb{E}^{\mathbb{Q}}\left[\mathrm{e}^{y D_{T}}\right]
$$

for arbitrary $x, y$. Using the tower property we have

$$
\mathbb{E}^{\mathbb{Q}}\left[\mathrm{e}^{x \beta_{t T}+y D_{T}}\right]=\mathbb{E}^{\mathbb{Q}}\left[\mathrm{e}^{x \xi_{t}} \mathbb{E}_{t}^{\mathbb{Q}}\left[\mathrm{e}^{\left(y-x \int_{0}^{t} \sigma_{s} \mathrm{~d} s\right) D_{T}}\right]\right],
$$

where we have inserted the definition of $\beta_{t T}$ given in the statement of the Proposition. We consider the inner expectation first. From equation (73) for the conditional expectation of a function of $D_{T}$ we deduce that

$$
\mathbb{E}_{t}^{\mathbb{Q}}\left[\mathrm{e}^{\left(y-x \int_{0}^{t} \sigma_{s} \mathrm{~d} s\right) D_{T}}\right]=\Phi_{t}^{-1} \int_{0}^{\infty} p(z) \mathrm{e}^{\left(y-x \int_{0}^{t} \sigma_{s} \mathrm{~d} s\right) z} \mathrm{e}^{z \int_{0}^{t} \nu_{u} \mathrm{~d} W_{u}^{*}-\frac{1}{2} z^{2} \int_{0}^{t} \nu_{u}^{2} \mathrm{~d} u} \mathrm{~d} z,
$$

where the process $\left\{\Phi_{t}\right\}$ is defined by (112). We now change the probability measure from $\mathbb{Q}$ to $\mathbb{B}$, so that the term $\Phi_{t}^{-1}$ appearing in (130) drops out to give us

$$
\begin{aligned}
\mathbb{E}^{\mathbb{Q}}\left[\mathrm{e}^{x \xi_{t}}\right. & \left.\mathbb{E}_{t}^{\mathbb{Q}}\left[\mathrm{e}^{\left(y-x \int_{0}^{t} \sigma_{s} \mathrm{~d} s\right) D_{T}}\right]\right] \\
& =\mathbb{E}^{\mathbb{B}}\left[\mathrm{e}^{x \xi_{t}} \int_{0}^{\infty} p(z) \mathrm{e}^{\left(y-x \int_{0}^{t} \sigma_{s} \mathrm{~d} s\right) z} \mathrm{e}^{z \int_{0}^{t} \nu_{u} \mathrm{~d} W_{u}^{*}-\frac{1}{2} z^{2} \int_{0}^{t} \nu_{u}^{2} \mathrm{~d} u} \mathrm{~d} z\right] \\
& =\int_{0}^{\infty} p(z) \mathbb{E}^{\mathbb{B}}\left[\mathrm{e}^{x(T-t) \int_{0}^{t} \frac{1}{T-s} \mathrm{~d} W_{s}^{*}+\left(y-x \int_{0}^{t} \sigma_{s} \mathrm{~d} s\right) z+z \int_{0}^{t} \nu_{s} \mathrm{~d} W_{s}^{*}-\frac{1}{2} z^{2} \int_{0}^{t} \nu_{s}^{2} \mathrm{~d} s}\right] \mathrm{d} z \\
& =\int_{0}^{\infty} p(z) \mathrm{e}^{\left(y-x \int_{0}^{t} \sigma_{s} \mathrm{~d} s\right) z-\frac{1}{2} z^{2} \int_{0}^{t} \nu_{s}^{2} \mathrm{~d} s+\frac{1}{2} \int_{0}^{t} \alpha_{s}^{2} \mathrm{~d} s} \mathbb{E}^{\mathbb{B}}\left[\mathrm{e}^{\int_{0}^{t} \alpha_{s} \mathrm{~d} W_{s}^{*}-\frac{1}{2} \int_{0}^{t} \alpha_{s}^{2} \mathrm{~d} s}\right] \mathrm{d} z
\end{aligned}
$$


where $\alpha_{s}=x(T-t) /(T-s)+z \nu_{s}$, and therefore

$$
\mathbb{E}^{\mathbb{Q}}\left[\mathrm{e}^{x \beta_{t T}+y D_{T}}\right]=\int_{0}^{\infty} p(z) \mathrm{e}^{\left(y-x \int_{0}^{t} \sigma_{s} \mathrm{~d} s\right) z-\frac{1}{2} z^{2} \int_{0}^{t} \nu_{s}^{2} \mathrm{~d} s+\frac{1}{2} \int_{0}^{t} \alpha_{s}^{2} \mathrm{~d} s} \mathrm{~d} z
$$

Furthermore, making use of relation (76) we have

$$
\exp \left(-x z \int_{0}^{t} \sigma_{s} \mathrm{~d} s-\frac{1}{2} z^{2} \int_{0}^{t} \nu_{s}^{2} \mathrm{~d} s+\frac{1}{2} \int_{0}^{t} \alpha_{s}^{2} \mathrm{~d} s\right)=\exp \left(\frac{t(T-t)}{2 T} x^{2}\right) .
$$

As a consequence, it follows from (132) that

$$
\mathbb{E}^{\mathbb{Q}}\left[\mathrm{e}^{x \beta_{t T}+y D_{T}}\right]=\left(\int_{0}^{\infty} p(z) \mathrm{e}^{y z} \mathrm{~d} z\right) \exp \left(\frac{t(T-t)}{2 T} x^{2}\right) .
$$

This establishes the independence of $\left\{\beta_{t T}\right\}$ and $D_{T}$.

The factorisation (134) also shows that the process $\left\{\beta_{t T}\right\}$ is $\mathbb{Q}$-Gaussian, with mean zero and variance $t(T-t) / T$. To establish that $\left\{\beta_{t T}\right\}$ is a Brownian bridge, we must show that for $s \leq t$ the covariance of $\beta_{s T}$ and $\beta_{t T}$ is given by $s(T-t) / T$. Alternatively, it suffices to analyse the moment generating function $\mathbb{E}\left[\mathrm{e}^{x \beta_{s T}+y \beta_{t T}}\right]$. We proceed as follows. First, using the tower property we have

$$
\begin{aligned}
\mathbb{E}^{\mathbb{Q}}\left[\mathrm{e}^{x \beta_{s T}+y \beta_{t T}}\right] & =\mathbb{E}\left[\mathrm{e}^{x \xi_{s}+y \xi_{t}-\left(x \int_{0}^{s} \sigma_{u} \mathrm{~d} u+y \int_{0}^{t} \sigma_{u} \mathrm{~d} u\right) D_{T}}\right] \\
& =\mathbb{E}\left[\mathrm{e}^{x \xi_{s}+y \xi_{t}} \mathbb{E}_{t}^{\mathbb{Q}}\left[\mathrm{e}^{-\left(x \int_{0}^{s} \sigma_{u} \mathrm{~d} u+y \int_{0}^{t} \sigma_{u} \mathrm{~d} u\right) D_{T}}\right]\right] .
\end{aligned}
$$

Next, by use of formula (73), the inner expectation can be carried out to give

$$
\mathbb{E}^{\mathbb{Q}}\left[\mathrm{e}^{x \beta_{s}+y \beta_{t T}}\right]=\mathbb{E}\left[\mathrm{e}^{x \xi_{s}+y \xi_{t}} \Phi_{t}^{-1} \int_{0}^{\infty} p(z) \mathrm{e}^{-\left(x \int_{0}^{s} \sigma_{u} \mathrm{~d} u+y \int_{0}^{t} \sigma_{u} \mathrm{~d} u\right) z} \mathrm{e}^{z \int_{0}^{t} \nu_{u} \mathrm{~d} W_{u}^{*}-\frac{1}{2} z^{2} \int_{0}^{t} \nu_{u}^{2} \mathrm{~d} u} \mathrm{~d} z\right]
$$

If we change the probability measure to $\mathbb{B}$ the random variable $\Phi_{t}$ in the denominator drops out, and we have

$$
\mathbb{E}^{\mathbb{Q}}\left[\mathrm{e}^{x \beta_{s}+y \beta_{t T}}\right]=\int_{0}^{\infty} p(z) \mathrm{e}^{-\left(x \int_{0}^{s} \sigma_{u} \mathrm{~d} u+y \int_{0}^{t} \sigma_{u} \mathrm{~d} u\right) z-\frac{1}{2} z^{2} \int_{0}^{t} \nu_{u}^{2} \mathrm{~d} u} \mathbb{E}^{\mathbb{B}}\left[\mathrm{e}^{x \xi_{s}+y \xi_{t}+z \int_{0}^{t} \nu_{s} \mathrm{~d} W_{s}^{*}}\right] \mathrm{d} z .
$$

Let us consider the inner expectation first. By defining $a_{u}=x(T-s) /(T-u)$ and $b_{u}=$ $y(T-t) /(T-u)+z \nu_{u}$ we can write

$$
\mathbb{E}^{\mathbb{B}}\left[\mathrm{e}^{x \xi_{s}+y \xi_{t}+z \int_{0}^{t} \nu_{s} \mathrm{~d} W_{s}^{*}}\right]=\mathbb{E}^{\mathbb{B}}\left[\mathrm{e}^{\int_{0}^{s} a_{u} \mathrm{~d} W_{u}^{*}+\int_{0}^{t} b_{u} \mathrm{~d} W_{u}^{*}}\right] .
$$

However, since $\left\{W_{t}^{*}\right\}$ is a $\mathbb{B}$-Brownian motion, using the properties of Gaussian random variable we find that

$$
\mathbb{E}^{\mathbb{B}}\left[\mathrm{e}^{\int_{0}^{s} a_{u} \mathrm{~d} W_{u}^{*}+\int_{0}^{t} b_{u} \mathrm{~d} W_{u}^{*}}\right]=\exp \left\{\frac{1}{2}\left(\int_{0}^{s} a_{u}^{2} \mathrm{~d} u+\int_{0}^{t} b_{u}^{2} \mathrm{~d} u+2 \int_{0}^{s} a_{u} b_{u} \mathrm{~d} u\right)\right\} .
$$

Substituting the definitions of $\left\{a_{u}\right\}$ and $\left\{b_{u}\right\}$ into the right side of (139) and combining the result with the remaining terms in the exponent of the right side of (137) we find that the 
terms involving the integration variable $z$ drop out, and we are left with the integral of the density function $p(z)$, which is unity. Gathering the remaining terms we obtain

$$
\mathbb{E}^{\mathbb{Q}}\left[\mathrm{e}^{x \beta_{s T}+y \beta_{t T}}\right]=\exp \left\{\frac{1}{2}\left(x^{2} \frac{s(T-s)}{T}+y^{2} \frac{t(T-t)}{T}+2 x y \frac{s(T-t)}{T}\right)\right\} .
$$

It follows that the covariance of $\beta_{s T}$ and $\beta_{t T}$ for $s \leq t$ is given by

$$
\left.\frac{\partial^{2}}{\partial x \partial y} \mathbb{E}^{\mathbb{Q}}\left[\mathrm{e}^{x \beta_{s T}+y \beta_{t T}}\right]\right|_{x=y=0}=\frac{s(T-t)}{T} .
$$

This establishes the assertion that $\left\{\beta_{t T}\right\}$ is a $\mathbb{Q}$-Brownian bridge.

The result above shows that, for the class of price processes we are considering, even if at the outset we take the "usual" point of view in financial modelling, and regard the price process of the asset as being adapted to some "prespecified" filtration, nevertheless it is possible to deduce the structure of the underlying information-based model.

\section{MULTI-FACTOR MODELS WITH A TIME-DEPENDENT INFORMATION FLOW RATE}

Let us now turn to consider the case of a single cash flow $D_{T}$ that depends on a multiplicity of market factors $\left\{X_{T_{k}}^{\alpha}\right\}_{k=1, \ldots, n}^{\alpha=1, \ldots, m_{k}}$, where we have the $n$ pre-designated information dates $\left\{T_{k}\right\}_{k=1,2, \ldots, n}$, and where for each value of $k$ we have a set of $m_{k}$ market factors. For simplicity we set $T=T_{n}$. Each market factor $X_{T_{k}}^{\alpha}$ is associated with an information process

$$
\xi_{t T_{k}}^{\alpha}=X_{T_{k}}^{\alpha} \int_{0}^{t} \sigma_{s T_{k}}^{\alpha} \mathrm{d} s+\beta_{t T_{k}}^{\alpha}
$$

where $X_{T_{k}}^{\alpha}$ and $\left\{\beta_{t T_{k}}^{\alpha}\right\}$ are independent. It should be evident that although the random variable $D_{T}$ representing the cash flow is $\mathcal{F}_{T}$-measurable, the values of some of the $X$-factors upon which it depends may be revealed at earlier times. That is to say, the uncertainties arising from some of the economic elements affecting the value of the cash flow at time $T$ may be resolved before that time.

Since the $X$-factors are independent, it follows that for each market factor the associated conditional density process $\pi_{t T_{k}}^{\alpha}(x)$ takes the form given in (66), and the corresponding dynamical equation is given by

$$
\mathrm{d} \pi_{t T_{k}}^{\alpha}=\nu_{t T_{k}}^{\alpha}\left(x_{k}^{\alpha}-\mathbb{E}^{\mathbb{Q}}\left[X_{T_{k}}^{\alpha} \mid \mathcal{F}_{t}\right]\right) \pi_{t T_{k}}^{\alpha} \mathrm{d} W_{t}^{\alpha k} .
$$

The function $\nu_{t T_{k}}^{\alpha}$ appearing here is given by an expression of the form (69):

$$
\nu_{t T_{k}}^{\alpha}=\sigma_{t T_{k}}^{\alpha}+\frac{1}{T_{k}-t} \int_{0}^{t} \sigma_{s T_{k}}^{\alpha} \mathrm{d} s .
$$

The innovation process $\left\{W_{t}^{\alpha k}\right\}$ is defined in terms of $\left\{\xi_{t T_{k}}^{\alpha}\right\}$ via a relation of the form

$$
W_{t}^{\alpha k}=\xi_{t T_{k}}^{\alpha}+\int_{0}^{t} \frac{1}{T_{k}-s} \xi_{s T_{k}}^{\alpha} \mathrm{d} s-\int_{0}^{t} \nu_{s T_{k}}^{\alpha} X_{T_{k}}^{\alpha} \mathrm{d} s .
$$


The conditional expectation $\mathbb{E}^{\mathbb{Q}}\left[D_{T} \mid \mathcal{F}_{t}\right]$ is thus given by the multi-dimensional integral

$$
\begin{aligned}
D_{t T}= & \int_{0}^{\infty} \cdots \int_{0}^{\infty} \Delta_{T}\left(x_{1}^{1}, \ldots, x_{1}^{m_{1}}, \ldots, x_{n}^{1}, \ldots, x_{n}^{m_{n}}\right) \\
& \times \pi_{t 1}\left(x_{1}^{1}\right) \cdots \pi_{t 1}\left(x_{1}^{m_{1}}\right) \cdots \pi_{t n}\left(x_{n}^{1}\right) \cdots \pi_{t n}\left(x_{n}^{m_{n}}\right) \mathrm{d} x_{1}^{1} \cdots \mathrm{d} x_{1}^{m_{1}} \cdots \mathrm{d} x_{n}^{1} \cdots \mathrm{d} x_{n}^{m_{n}},
\end{aligned}
$$

and the price of the asset for $t<T$ is $S_{t}=P_{t T} D_{t T}$. A straightforward application of Ito's rule then establishes the following result:

Proposition 7 . The price process $\left\{S_{t}\right\}_{0 \leq t<T}$ of an asset that pays a single dividend $D_{T}$ at time $T\left(=T_{n}\right)$ depending on the market factors $\left\{X_{T_{k}}^{\alpha}\right\}_{k=1,2, \ldots, m_{k}}^{\alpha=1,2, \ldots}$, satisfies the dynamical equation

$$
\mathrm{d} S_{t}=r_{t} S_{t} \mathrm{~d} t+\sum_{k=1}^{n} \sum_{\alpha=1}^{m_{k}} \nu_{t T_{k}}^{\alpha} \operatorname{Cov}_{t}\left[D_{T}, X_{T_{k}}^{\alpha}\right] \mathrm{d} W_{t}^{\alpha k}
$$

where

$$
D_{T}=\Delta_{T}\left(X_{T_{1}}^{\alpha}, \ldots, X_{T_{k}}^{\alpha}\right) .
$$

Here $\operatorname{Cov}_{t}\left[D_{T}, X_{T_{k}}^{\alpha}\right]$ denotes the covariance between the cash-flow $D_{T}$ and the market factor $X_{T_{k}}^{\alpha}$, conditional on the information $\mathcal{F}_{t}$ generated by the information processes $\left\{\xi_{s T_{k}}^{\alpha}\right\}_{0 \leq s \leq t}$.

In the more general case of an asset that pays multiple dividends (see Section VIII) the price is given by

$$
S_{t}=\sum_{k=1}^{n} \mathbb{1}_{\left\{t<T_{k}\right\}} P_{t T_{k}} \mathbb{E}^{\mathbb{Q}}\left[\Delta_{T_{k}}\left(\left\{X_{T_{j}}^{\alpha}\right\}_{j=1, \ldots, k}^{\alpha=1,2, \ldots, m_{j}}\right) \mid \mathcal{F}_{t}\right] .
$$

Proposition 8. The price process $\left\{S_{t}\right\}$ of an asset that pays the random dividends $D_{T_{k}}$ on the dates $T_{k}(k=1, \ldots, n)$ satisfies the dynamical equation

$$
\mathrm{d} S_{t}=r_{t} S_{t} \mathrm{~d} t+\sum_{k=1}^{n} \sum_{\alpha=1}^{m_{k}} \mathbb{1}_{\left\{t<T_{k}\right\}} \nu_{t T_{k}}^{\alpha} \operatorname{Cov}_{t}\left[D_{T_{k}}, X_{T_{k}}^{\alpha}\right] \mathrm{d} W_{t}^{\alpha k}+\Delta_{T_{k}} \mathrm{~d} \mathbb{1}_{\{t<T\}},
$$

where

$$
D_{T_{k}}=\Delta_{T_{k}}\left(\left\{X_{T_{j}}^{\alpha}\right\}_{j=1, \ldots, k}^{\alpha=1, \ldots, m_{j}}\right) .
$$

Here $\operatorname{Cov}_{t}\left[D_{T_{k}}, X_{T_{k}}^{\alpha}\right]$ denotes the covariance between the dividend $D_{T_{k}}$ and the market factor $X_{T_{k}}^{\alpha}$, conditional on the market information $\mathcal{F}_{t}$.

We conclude that the multi-factor, multi-dividend situation is fully tractable when the information-flow rates associated with the various market factors are time dependent. A straightforward extension of Proposition 8 allows us to formulate the joint price dynamics of a system of assets, the associated dividend flows of which may depend on common market factors. As a consequence, a specific model for stochastic volatility and correlation emerges for such a system of assets, and it is one of the main conclusions of this paper that such 
a model can be formulated. The information-based " $X$-factor" approach presented here thus offers a new insights into the nature of volatility and correlation, and as such may find applications in a number of different areas of financial risk analysis. We have in mind, in particular, applications to equity portfolios, credit portfolios, and insurance, all of which exhibit intertemporal market correlation effects. We also have in mind the problem of firmwide risk management and optimal capital allocation for banking institutions.

Acknowledgements. The authors thank T. Bielecki, T. Björk, I. Buckley, H. Bühlmann, S. Carter, I. Constantinou, M. Davis, J. Dear, A. Elizalde, B. Flesaker, H. Geman, V. Henderson, D. Hobson, T. Hurd, M. Jeanblanc, A. Lokka, J. Mao, B. Meister, M. Monoyios, M. Pistorius, M. Rutkowski, D. Taylor, and M. Zervos for stimulating discussions. The authors are also grateful for helpful comments made by seminar participants at various meetings where parts of this work have been presented, including: the Developments in Quantitative Finance conference, July 2005, Isaac Newton Institute, Cambridge; the Mathematics in Finance conference, August 2005, Kruger National Park, RSA; the School of Computational and Applied Mathematics, University of the Witwatersrand, RSA, August 2005; CEMFI (Centro de Estudios Monetarios y Financieros), Madrid, October 2005; the Department of Actuarial Mathematics and Statistics, Heriot-Watt University, December 2005; the Department of Mathematics, King's College London, December 2005; the Bank of Japan, Tokyo, December 2005; and Nomura Securities, Tokyo, December 2005. DCB acknowledges support from The Royal Society; LPH and AM acknowledge support from EPSRC (grant number GR/S22998/01); AM thanks the Public Education Authority of the Canton of Bern, Switzerland, and the UK Universities ORS scheme, for support.

\section{References.}

[1] K. Back, "Insider trading in continuous time", Rev. Fin. Studies 5, 387-407 (1992).

[2] K. Back and S. Baruch, "Information in securities markets: Kyle meets Glosten and Milgrom", Econometrica 72, 433-465 (2004).

[3] D. C. Brody, L. P. Hughston, and A. Macrina, "Beyond hazard rates: a new framework for credit-risk modelling", in Advances in Mathematical Finance: Festschrift Volume in Honour of Dilip Madan (Basel: Birkhäuser, 2007).

[4] R. S. Bucy and P. D. Joseph, Filtering for stochastic processes with applications to guidance (New York: Interscience Publishers, 1968).

[5] U. Cetin, R. Jarrow, P. Protter, and Y. Yildrim, "Modelling Credit Risk with Partial Information", Ann. Appl. Prob. 14, 1167-1172 (2004).

[6] M. H. A. Davis, "Complete-market models of stochastic volatility" Proc. Roy. Soc. Lond. A460, 11-26 (2004)

[7] M. H. A. Davis and S. I. Marcus, "An introduction to nonlinear filtering" in Stochastic systems: The mathematics of filtering and identification and application, M. Hazewinkel and J. C. Willems, eds. (Dordrecht: D. Reidel, 1981).

[8] D. Duffie and D. Lando, "Term structure of credit spreads with incomplete accounting information" Econometrica 69, 633-664 (2001). 
[9] M. Fujisaki, G. Kallianpur, and H. Kunita "Stochastic differential equations for the non linear filtering problem" Osaka J. Math. 9, 19 (1972).

[10] K. Giesecke, "Correlated default with incomplete information" J. Banking and Finance 28, 1521-1545 (2994).

[11] K. Giesecke and L. R. Goldberg, "Sequential default and incomplete information" $J$. Risk 7, 1-26 (2004).

[12] S. L. Heston, "A closed-form solution for options with stochastic volatility with applications to bond and currency options" Rev. Financial Studies 6, 327-343 (1993).

[13] X. Guo, R. A. Jarrow and Y. Zeng, "Information reduction in credit risk modelling" working paper (2005).

[14] R. A. Jarrow and P. Protter, "Structural versus reduced form models: a new information based perspective" J. Investment Management 2, 34-43 (2004).

[15] G. Kallianpur and C. Striebel, "Estimation of stochastic systems: Arbitrary system process with additive white noise observation errors" Ann. Math. Statist. 39, 785 (1968).

[16] I. Karatzas and S. E. Shreve, Brownian motion and stochastic calculus (Berlin: Springer, 1997).

[17] V. Krishnan, Nonlinear Filtering and Smoothing (New York: Dover, 2005).

[18] R. S. Liptser and A. N. Shiryaev, Statistics of Random Processes Vols. I and II, 2nd ed. (Berlin: Springer, 2000).

[19] A. Macrina, "An information-based framework for asset pricing: $X$-factor theory and its applications" PhD thesis, King's College London (2006).

[20] M. O'Hara, Market Microstructure Theory (Cambridge, Massachusetts: Blackwell, 1995).

[21] P. Protter Stochastic Integration and Differential Equations: A New Approach, 2nd ed. (Berlin: Springer, 2003).

[22] M. Rutkowski and N. Yu, "On the Brody-Hughston-Macrina approach to modelling of defaultable term structure", working paper, School of Mathematics, University of New South Wales, Sydney, downloadable at www.defaultrisk.com (2005).

[23] W. M. Wonham, "Some applications of stochastic differential equations to optimal nonlinear filtering" J. SIAM A2, 347 (1965).

[24] M. Yor, Some Aspects of Brownian Motion, Part I: Some Special Functionals (Basel: Birkhäuser, 1992).

[25] M. Yor, Some Aspects of Brownian Motion, Part II: Some Recent Martingale Problems (Basel: Birkhäuser, 1996). 